\title{
XII ISIN Conference on Immunonutrition
}

July 14-16, 2021 (Online): Abstracts

\section{Abstracts}

Guest Editors

Ascension Marcos, Madrid. Spain

Margarida Castell, Barcelona, Spain

Francisco J. Pérez Cano, Barcelona, Spain

\section{Contents}

2 Abstracts

34 Author Index

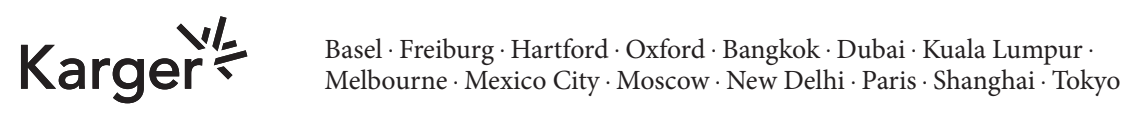




\section{Nutrition \& Metabolism}

\section{$451 / 9$ \\ Effects of Parental Nutrition on the Immune System in Head and Neck Cancer Patients \\ Candiloro, F. \\ Santa Lucia Foundation}

Head and neck squamous cell carcinoma (HNSCC) patients often face malnutrition. Malnutrition is associated with immunosuppression (1) and improving the nutritional status with parenteral nutrition (PN) can restore immune competence (2). The bags for PN contain polyunsaturated fatty acids (PUFAs) that influence the immune system (3). The guidelines suggest caution in the use lipid emulsions of $\omega 6$ in patients with uncontrolled inflammatory responses, these may instead be advisable in patients unable to develop an adequate antitumoral response. We studied the effects of PUFAs in cultures of PBMCs. We also determined the effects of PN (olive oil-based PN and fish oil-based PN) on the immune responses. PUFAs may modulate the activation of immune cell subsets, and different cells respond to distinct lipid. We found that the composition of the bags for $\mathrm{PN}$ influence the immune response. One-week of OO-based PN was sufficient to affect the functional response of immune cells.

We found that patients treated with OO show a decrease in the frequency of Tregs cells after OO-treatment. These data point to a positive effect on the antitumoral immune response induced by OO-based PN.

Acknowledgments: Thanks to all the patients.

Financial Support: This work was partially supported by Baxter.

Conflict of Interest: THE AUTHORS DON'T DECLARE CONFLICTS OF INTEREST.

Authorship: FC, MP performed the experiments GB, LB wrote the manuscript and VB, RC enrolled the patients.

Keywords: Fatty acids, parenteral nutrition, anticancer immunity.

\section{References}

1 Moy JD, Biological mechanisms of immune escape and implications for immunotherapy in head and neck squamous cell carcinoma. European Journal of Cancer (2017) doi: 10.1016/j.ejca.2016.12.035.

2 Jin Y, Effects of Post-Surgical Parenteral Nutrition on Patients with Gastric Cancer.Cell Physiol Biochem (2018) doi:10.1159/000493410.

3 Harbige LS Fatty acids, the immune response, and autoimmunity: a question of n- 6 essentiality and the balance between n- 6 and n-3. Lipids (2003).

\section{$451 / 10$ \\ Nutritional and Biological Action of Polyphenol-Extracts from Colombian Passiflora Ligularis Juss: In Vivo Study to Evaluate Weight Gain and Inflammation Control}

Restrepo-López, JP. 1; Ángel-Isaza, J. '; Narváez-Solarte, W. ${ }^{1}$; González-Correa, CH. ; Liu, J. ${ }^{2}$; Carmona-Hernández, JC. ${ }^{1}$

${ }^{1}$ Nutrition, Metabolism and Food Safety Research Group. University of Caldas. Manizales. Colombia; ${ }^{2}$ Department of Medicine. Harvard Universit. Boston. USA

Inflammation is a state underlying diseases like cardiovascular disease and obesity affecting millions of people worldwide $(1,2)$. Passifloras are reported to offer multiple health benefits (3). The objective of this study was to evaluate the effect of the polyphenolrich pulp and seed extract of Passiflora ligularis Juss (Colombian granadilla, sweet passion-fruit) on the risk of low-grade chronic inflammation associated with a high-fat diet. Albino mice distributed in 3 treatments $(n=36)$ with a control group, a high-fat diet and a group with a high-fat diet along with supplementation with $3.0 \mathrm{~g} / \mathrm{L}$ of granadilla polyphenol-extract. Consumption of food and water were constantly registered. Weight gain and inflammatory markers TNF- $\alpha$ and IL- 6 were evaluated. The supplemented-granadilla-extract significantly reduced $(\mathrm{p}<0.05)$ weight gain and TNF- $\alpha$ in comparison with the animals that received the high-fat diet without polyphenol supplementation; values for IL-6 were not affected. These results indicate that polyphenols in Passiflora ligularis Juss contribute to regulating weight gain and to tackling inflammatory conditions.

Acknowledgments: Colciencias (Bogota-Colombia), Universties de Manizales and Caldas (Manizales-Colombia).

Financial Support: Colciencias, Universidad de Manizales.

Conflict of Interest: None.

Authorship: JA, WN, CHG, JCC: planning the procedures, obtaining financial assistance. JPR, JA, JCC: methodological proceedings. WN, JL, CHG: laboratory procedures. All authors: editing.

Keywords: Passiflora ligularis Juss; polyphenols; granadilla; overweight; inflammation; interleukins.

\section{References}

1 Rani V, et al (2016) Oxidative stress and metabolic disorders... Life Sci $148,183-193$.

2 Esser N, et al (2014) Inflammation as a link between obesity, ...Res Clin Pr 105, 141-50.

3 Saravanan S, Parimelazhagan T. (2014) In vitro antioxidant, ...Passiflora ligularis Juss. FSHW. 3, 56-64. 
451/11

\section{Activated Intestinal Epithelial Cells Conditioned with 2'-Fucosyllactose and CpG-ODN Instruct moDC to Drive Th1 Differentiation}

\author{
Ayechu-Muruzabal, V. '; Overbeek, S. ${ }^{2}$; Kostadinova, A. ${ }^{2}$; \\ Garssen, J. ${ }^{2}$; van't Land, B. ${ }^{3}$; Willemsen, L. ${ }^{4}$
}

${ }^{1}$ Division of Pharmacology. Utrecht Institute for Pharmaceutical Sciences. Utrecht University; ${ }^{2}$ Department of Immunology. Danone Nutricia Research B.V. Utrecht. The Netherlands; ${ }^{3}$ Center for Translational Immunology. Wilhelmina Children's Hospital. University Medical Center Utrecht; ${ }^{4}$ Division of Pharmacology.

Utrecht Institute for Pharmaceutical Sciences. Utrecht University

This study aims to investigate the immunomodulatory properties of 2'-fucosyllactose (2'-FL) using a co-culture model developed to study the crosstalk between intestinal epithelial cells (IEC) and innate and adaptive immune cells. IECs, co-cultured with aCD3/ CD28-activated peripheral blood mononuclear cells (PBMC), were apically exposed to NDOs and CpG, washed and co-cultured with immature monocyte derived dendritic cells (moDC). Subsequently, moDC were co-cultured with naïve CD4+ T-cells. In the presence of CpG, both 2'-FL- or GF-exposed IEC enhanced Th1 type IFNgamma and regulatory IL-10 secretion of PBMCs, compared to $\mathrm{CpG}$ alone, while Th2 type IL-13 was reduced. MoDCs exposed to 2'-FL and CpG-conditioned IEC instructed IFNgamma and IL-10 secretion by CD4+ T-cells, suggesting the development of a regulatory Th1 response. These results reveal that 2'-FL and GF could contribute to the mucosal immune development by supporting the effect of microbial CpG DNA.

Acknowledgments: The authors acknowledge Ling Xiao and Nienke Kettelarij for the useful discussions and technical support.

Financial Support: This research was funded by Danone/ Nutricia Research B.V.

Conflict of Interest: None of the authors have a competing financial interest; JG is head of the division of Pharmacology, and partly employed by Nutricia Research. SO, AKand BL are employed by Nutricia Research. BL is leading a strategic alliance between University Medical Centre Utrecht/Wilhelmina Children's Hospital and Nutricia Research.

Authorship: Conceptualization, V.AM, B.L. and L.W.; investigation, V.AM.; writing and review, V.AM, S.O., A.K., J.G., B.L. and L.W.

Keywords: 2'-fucosyllactose, non-digestible oligosaccharides, intestinal epithelial cells, dendritic cells.

\section{Reference}

De Kivit, S. et al. (2013). J. Innate Immunity, 5(6), 625-638.

\section{1/12}

Activation of Murine Bone Marrow Derived Dendritic Cells by Soy Protein is Dependent on its Origin and Mouse Strain

Zhao, X. ${ }^{1}$; Knippels, L. ${ }^{2}$; Chen, H. $^{3}$; Garssen, J. ${ }^{4}$; Hogenkamp, A. ${ }^{1}$

${ }^{1}$ Utrecht University; ${ }^{2}$ Danone Nutricia Research; ${ }^{3}$ Nanchang

University; ${ }^{4}$ Danone Nutricia Research. Utrecht and Division

of Pharmacology Utrecht University. The Netherlands

Soybean is one of the most significant food allergies. At least 34 allergenic proteins involved in IgE binding have been identified in soybean, including subunits of $\beta$-conglycinin (7S), and the acidic and basic chains of glycinin (11S). We investigated whether exposure to crude soybean protein isolate (SPI), 7S and 11S isolated from soybean obtained from the USA or China would generate different responses in murine bone marrow derived dendritic cells (BMDC). To assess differences in BMDC activation, cells were exposed to soy proteins and stimulated with LPS. BMDC cell surface marker expression of CD40 and CD86 was determined, and cytokine expression of IL-12p20, IL-4, IL-10, IL-6 was determined. Soy protein from China, especially SPI, increased LPS-induced BMDC expression of CD86 and CD40 compared to LPS-only stimulated cells, but also when compared to cells treated with proteins derived from soybean from the USA. This difference in DC-activation may suggest that there could be differences in allergenicity depending on the source of the soybean. Moreover, CD86 and CD40 expression was observed to be higher in BMDC derived from female Balb/c mice compared to $\mathrm{C} 3 \mathrm{H}$ after exposure to SPI. In follow-up experiments, we will assess how these in vitro findings correlate to in vivo immune outcomes.

Acknowledgments: The authors are grateful to Nutricia Research.

Financial Support: This work was funded by the Chinese Scholarship Council and Nutricia Research.

Conflict of Interest: LK is employed at Nutricia research as Science Director Allergy and as affiliate associate professor at Utrecht University; JG is employed at Nutricia research as Senior Director Immunology and as professor Immunopharmacology at Utrecht University.

Authorship: Xiaoli Zhao.

Keywords: Dendritic cells; soy allergy; SPI.

\section{References}

No 
$451 / 14$

Effects of Fish Oil Omega-3 Fatty Acid Supplementation on Cardiometabolic Biomarkers in Women with Polycystic Ovary Syndrome and Metabolic Syndrome

Mekki, K. ${ }^{1}$; Benharrats, L. ${ }^{2}$; Senouci, A. ${ }^{3}$

${ }^{1}$ Department of Biology. Faculty of Natural and Life Science. University Oran $1 ;{ }^{2}$ Faculty of Natural and Life ScienLaboratory of Clinical and Metabolic Nutrition. University Oran $1 ;{ }^{3}$ Faculty of Natural and Life ScienLaboratory of Clinical and Metabolic Nutrition. University Oran1, Algeria

Objective: We evaluated the effects of omega-3 polyunsaturated fatty-acid (PUFA) supplementation on lipid profile, lipid and protein peroxidation, antioxidant defense and inflammation in women with polycystic ovary syndrome (PCOS) and metabolic syndrome (MS).

Subjects: Sixty women ( $29 \pm 6$ years) were recruited between September 2018 and June 2019. The diagnosis of PCOS was based on Rotterdam criteria and that of MS on the NCEP ATP 111 criteria. The prevalence of MS components was (21.6\% insulin resistance, 98.33\% hypertriglyceridemia, 45\% low HDL-C, 56.66\% high blood pressure and $100 \%$ abdominal obesity).

Intervention: All women received nutritional counseling adapted to a Mediterranean diet. They have been asked to consume olive oil for seasonings, whole grains, legumes, vegetables and fruits, combined with daily physical activity. Women with PCOS and MS were randomized into 2 groups; a treated group $(\mathrm{T} ; \mathrm{n}=30)$ received for 6 weeks supplementation with omega-3 fish oil (180mg EPA and 120mg DHA/1g; Doppelherz, Germany) 3g/day, 30 women were assigned to control group (C).

Results: After 6 weeks of intervention, in (T) compared to (C) there was a significant decrease $(\mathrm{p}<0.05)$ in fasting blood glucose levels. TG, TC, HDL-C and LDL-C were unchanged. No significant decrease was observed for CRP concentrations. TBARS and carbonyls amounts were unchanged, however, a significant increase by $7 \%$ in thiols and by $12 \%$ in SOD activity was observed $(\mathrm{p}<0.05)$ in $(\mathrm{T})$ compared to $(\mathrm{C})$. Catalase activity was similar.

Conclusion: Omega 3 supplementation improves hyperglycemia and antioxidant defense in patients with SOPK and MS and may lead to decreased rates of cardiovascular complications.

Acknowledgments: WASSILA BENHABIB from Department of Epidemiology, University Hospital of Oran.

YAMINA ABED from Public Health Establishment of Proximity, Es Senia.

Financial Support: Doppelherz, Germany.

Conflict of Interest: No

Authorship: All authors contributed effectively to this study.

Keywords: Polycystic ovary syndrome, metabolic syndrome, Omega 3, Mediteranean diet, Inflammation, Oxidative stress, dyslipidemia.

\section{References}

Faghfoori et al. Diabetes Metab Syndr. 2017; 11 (Suppl1): S429-S432. Goth . Clin Chim Acta 1991; 196: 143-52.

Hallajzadeh et al. J Cardiovasc Thorac Res, 2018, 10(2), 56-69.

Hidalgo-Mora et al. Maturitas Volume 132, February 2020, p 65-69 Levin et al. Methods. Enzymol 1990. 186: 464-478.

Marklund et Marklund . Eur J Biochem. 1974 Sep 16;47(3):469-74.

Quintanilha et al. Biophysical Journal February 1982. 37(1):68-9.

Sedlak et Lindsay. Anal Biochem. 1968 Oct 24;25(1):192-205.

\section{1/16 \\ Lupine Peptides-Treatment Reduces Lipid Accumulation in Hypercholesterolemic Mice Model}

Santos Sánchez, G. ${ }^{1}$; Cruz Chamorro, I. '; Fernández Santos, J.2; Pedroche, J. ${ }^{3}$; Millán Linares, M. ${ }^{4}$; Carrillo Vico, A. ${ }^{1}$

${ }^{1}$ Instituto de Biomedicina de Sevilla. IBiS (Universidad de Sevilla, HUVR, Junta de Andalucía, CSIC); ${ }^{2}$ Departamento Citología e Histología Normal y Patológica. Universidad de Sevilla.; ${ }^{3}$ Plant Protein Group. Instituto de la Grasa. CSIC. Seville. Spain;

${ }^{4}$ Department of Food \& Health. Instituto de la grasa. CSIC

Non-alcoholic fatty liver disease (NAFLD) is the most principal cause of liver disease in Western countries. Liver fat accumulation, inflammation, and injury of liver cells are responsible for NAFLD (1). In addition, NAFLD is strongly linked to central abdominal obesity (2). Recently, our group demonstrated that lupine protein hydrolysates (LPHs) had lipid lowering, antioxidant, anti-inflammatory and anti-atherogenic effects in ApoE-/- mice, a preclinical model of hypercholesterolemia. Therefore, the objective of this study was to evaluate the possible effects of LPHs on hepatic steatosis and the accumulation of abdominal adipose tissue in ApoE/-mice. Thirty-four male mice fed with a high-fat diet were intragastrically treated with LPHs (or vehicle) for 12 weeks. Lipid accumulation in liver and adipose tissue were analysed. A significant decrease in steatosis was observed in LPHs-treated mice. In addition, LPHs reduced the gene expression of liver CD36, an important cholesterol scavenger receptor, in treated-mice. Moreover, LPHs-treated mice showed a reduction in the abdominal adipose tissue and less adipose hypertrophy in comparison to control group. We can conclude that LPHs palliate the generation of the steatotic liver caused by the intake of a high-fat diet and reduce abdominal obesity.

Acknowledgments: Beatriz Rodríguez-Ortiz, Drs. Nuria Álvarez-Sánchez, Patricia Judith Lardone, and Juan Miguel Guerrero.

Financial Support: Andalusian Government Ministry of Health [PC-0111-2016-0111]. G.S.-S. [FPU grant 16/02339].

Conflict of Interest: None.

Authorship: Design: A.C.-V., J.P, and MC.M.-L. Development: G.S.-S., I.C.-C., and JM.F.-S. Analysis: G.S.-S., I.C.-C., and JM.F.-S. Writing: G.S.-S., I.C.-C., and A.C.-V.

Keywords: Lupine hydrolysates, NAFLD, adipose tissue, ApoE, High-fat diet. 


\section{References}

1 Milić, S. World J Gastroenterol. 2014; 20(28), 9330.

2 Polyzos, S. Metabolism. 2019; 92, 82-97.

$451 / 17$

\section{Effect of Different Peruvian Cocoa Varieties Intake} in an Allergic Asthma Model in Rats

Périz, M. ${ }^{1}$; Massot-Cladera, M. ${ }^{1}$; Pastor-Soplin, S. ${ }^{2}$; Castell Escuer, M. ${ }^{1}$; Best, I. ; Pérez-Cano, F. ${ }^{1}$

${ }^{1}$ Physiology Section, Faculty of Pharmacy and Food Science, University of Barceloma, INSA-UB, Spain; ${ }^{2}$ Cacao Program; Forest Engineering Career, South Scientific University, Lima, Peru;

${ }^{3}$ South Scientific University, Lima, Peru

The study aimed to investigate the effects of three Peruvian cocoa varieties on the anaphylactic response in an allergic asthma rat model. For this purpose, female Brown Norway rats were distributed into five experimental groups: REF and A (healthy and asthmatic, respectively, with standard diet) and OC, APC and $\mathrm{CMC}$ in which the asthmatic animals received diets containing $10 \%$ cocoa paste either from an ordinary cocoa, "Amazonas Perú" or "Criollo de Montaña" varieties respectively. After one week of diet, asthma was induced by two intraperitoneal sensitizations of ovalbumin and an intranasal challenge. Anaphylaxis was then assessed by changes in the motor activity and body temperature. Moreover, specific IgE antibodies were evaluated in the bronchoalveolar lavage (BALF) and serum. The challenge decreased the motility and the body temperature in the group A even $20 \mathrm{~h}$ after the shock. Only the OC group showed a partial prevention of this anaphylactic reaction. Animals from group A developed antiOVA IgE antibodies both in BALF and serum. The levels of these antibodies were lower in the OC group in both compartments, whereas the CMC diet decreased their levels only in BALF. Overall, the diet enriched with the ordinary cocoa exerted the highest protective effects on the variables considered in this allergic asthma model.

Acknowledgments: The authors thank Dr. T. Cambras and the students involved for their technical assistance.

Financial Support: The present work was funded by the FONDECYT of the CONCYTEC.

Conflict of Interest: The authors declare no conflict of interest.

Authorship: MP, MM-C. and MC carried out them, analysed the data and wrote the abstract and SP-S, IA-B, and FP-C. reviewed it.

Keywords: Allergic asthma model, IgE, Peruvian cocoa.

\section{$451 / 19$ \\ Investigating the Impact of Dietary Emulsifiers on Intestinal Epithelial Cells Responses and Inflammation}

Saiz. G.; Melgar, S.; Fanning, A.

APC Microbiome. Cork. Ireland

Aim: This study aims to investigate three of the most common emulsifiers used in the food industry, polysorbate-80, carboxymethylcellulose, and lecithin, on the function(s) in two human intestinal epithelial cell (IEC) lines.

Inflammatory Bowel Diseases (IBD) is a chronic inflammatory condition that has become pandemic worldwide, with more than five million people affected in the developed countries[1]. Two factors linked to this condition are the diet and intestinal microbiota. High fat diets (rich in fat/low in fibres) and processed food are risk factors for IBD [3]. Emulsifiers are one of the main additives used in the food industry. However, recent evidence in mice indicated that these emulsifiers can disturb the host-microbiota $[2,4]$.

Key findings: IEC cultured with the emulsifiers, especially polysorbate-80, provoke alterations in metabolism, proliferation, cell death and cytokine response. The alterations in gene expression was associated to autophagy, ER-stress and apoptosis pathways. In conclusion, our data suggest that these emulsifying agents might contribute to the increased incidence of chronic inflammatory conditions and metabolic syndrome by altering cell microbial responses.

Acknowledgments: The support from Dr Amanda Lohan and Lab 4.38 is highly acknowledged.

Financial Support: This study was supported by a research grant from SFI to APC Microbiome Ireland grant SFI/12/RC/2273_ P2 (S. Melgar) and a IRC scholarship, GOIPG/2019/4528 (G. Saiz).

\section{Conflict of Interest: None.}

Authorship: Gonzalo Saiz has performed the experimental work of the study, analysed the data and wrote the article. Aine Fanning provided training on the experimental work. Silvia Melgar is the supervisor of the study, developed the design and plan of the experiments.

Keywords: IBD, epithelial cells, inflammation, emulsifiers, cell death.

\section{References}

Chassaing B, et al. Nature; 519: 92-96 (2015).

2 Chassaing B, et al. Gut; 1-14 (2017).

3 Melgar S, et al. Front Immunol; 28;8:838 (2017).

4 Viennois E, et al. Cancer Res; 27-41 (2017).

\section{References}

N/A 


\section{$451 / 23$}

Effect of the Mediterranean Diet on Cardiometabolic Biomarkers in Women with Polycystic Ovary Syndrome

Mekki, K. ${ }^{1}$; Benharrats, L. ${ }^{2}$; Senouci, A. ${ }^{3}$

${ }^{1}$ Department of Biology. Faculty of Natural and Life Science. University Oran $1 ;{ }^{2}$ Faculty of Natural and Life ScienLaboratory of Clinical and Metabolic Nutrition. University Oran $1 ;{ }^{3}$ Faculty of Natural and Life ScienLaboratory of Clinical and Metabolic Nutrition. University Oran1, Algeria

Objective: To evaluate the effect of the Mediterranean diet advices on dietary behavior and metabolic biomarkers in patients with PCOS.

Methods: Study was carried in 102 patients $(30+7$ years) with PCOS. The diagnosis of PCOS was based on Rotterdam criteria. All women received nutritional counseling adapted to the Mediterranean diet. They have been asked to consume olive oil for seasonings, whole grains, legumes, vegetables and fruits, combined with daily physical activity. Dietary survey and biomarkers analysis were assessed at baseline (T0), after 3 weeks (T1) and 6 weeks (T2).

Results: After 6 weeks of intervention, compared to T0, there was a decrease in total energy intake by $(-33 \%)$ at $\mathrm{T} 1$ and $(-34 \%)$ at T2 $(\mathrm{p}<0.001)$. A T1 and T2 decrease $(\mathrm{p}<0.001)$ was noted in carbohydrates intake, in proteins and lipids. A significant increase in monounsaturated fatty acids intake at T1 and T2 $(\mathrm{p}<0.001)$.

Significant decrease in BMI by $(-4 \%)$ at $\mathrm{T} 1(\mathrm{p}<0.05)$ and $(-8 \%)$ at T2 $(\mathrm{p}<0.01)$, and in waist circumference by $(-5 \%)$ at $\mathrm{T} 1$ and $(-8 \%)$ at T2 $(\mathrm{p}<0.001)$. Glucose values decreased significantly at T1 $(\mathrm{p}<0.05)$ and $\mathrm{T} 2(\mathrm{p}<0.001)$. Total cholesterol decreased by $(-6 \%)$ at $\mathrm{T} 1(\mathrm{p}<0.05)$. HDL-C increased by $(11 \%)$ at T1 $(\mathrm{p}<0.001)$. A significant decrease was observed in TBARS by $(-34 \%)$ at T2 $(\mathrm{p}<0.01)$. $\mathrm{SOD}$, catalase activities and thiols amount increased at T2 $(\mathrm{p}<0.001)$.

Conclusion: Lifestyle modification based on the Mediterranean diet principles remains the most important therapeutic strategy in PCOS women and it can prevent overweight, insulin resistance and metabolic abnormalities reducing cardiovascular risk.

Acknowledgments: WASSILA BENHABIB from Department of Epidemiology, University Hospital of Oran YAMINA ABED from Public Health Establishment of Proximity, Es Senia.

Financial Support: ATRSS.

Conflict of Interest: No

Authorship: All authors contributed effectively to the study. Keywords: Mediterranean diet, lifestyle, PCOS, Cardiometabolic risk.

\section{References}

\section{$451 / 24$ \\ Breastfeeding Promotes Early Neonatal Regulatory T Cell Expansion and Immune Tolerance of Non-Inherited Maternal Antigens}

Toldi, G.

University of Birmingham, Birmingham, United Kingdom

Background: Breastfeeding is associated with long-term health benefits, such as a lower incidence of childhood infections, asthma, obesity and autoimmune disorders. However, little is known regarding how the maternal and neonatal immune systems interact after parturition when the neonate receives nutrition from maternal breastmilk.

Methods: We undertook a comparative analysis of immune repertoire and function at birth and 3 weeks of age in a cohort of 38 term neonates born by caesarean section grouped according to feeding method (breastmilk versus formula). We used flow cytometry to study the immune phenotype in neonatal and maternal blood samples and mixed lymphocyte reactions to establish the proliferation response of neonatal versus maternal lymphocytes and vice versa. The microbiome of neonatal stool samples was also investigated using 16S rRNA sequencing.

Results: We show that the proportion of regulatory $\mathrm{T}$ cells (Tregs) increases in this period and is nearly two-fold higher in exclusively breastfed neonates compared to those who received formula milk only. Moreover, breastfed neonates show a specific and Treg-dependent reduction in proliferative $\mathrm{T}$ cell responses to non-inherited maternal antigens (NIMA), associated with a reduction in inflammatory cytokine production. We also observed the enrichment of short chain fatty acid producing taxa (Veillonella and Gemella) in stool samples of exclusively breastfed neonates.

Conclusions: These findings add to our understanding of mechanisms by which early life nutrition can determine long term health outcomes. Our data indicate that exposure of the neonate to maternal cells through breastfeeding acts to drive the maturation of Tregs and 'tolerizes' the neonate towards NIMA.

Acknowledgments: Hannah Wood, Animesh Acharjee, Hayden Pearce, Mohammed Quraishi, Richard Powell, Amanda Rossiter, Andrew Beggs, Andrew Ewer, Paul Moss.

Financial Support: N/A.

Conflict of Interest: N/A.

Authorship: N/A.

Keywords: Breastfeeding, microbiome, neonate, non-inherited maternal antigen, regulatory $\mathrm{T}$ cell, Th17.

\section{References}

N/A

Levin et al. Methods. Enzymol 1990. 186: 464-478.

Marklund et Marklund. Eur J Biochem. 1974 Sep 16;47(3):469-74.

Quintanilha et al. Biophysical Journal February 1982.37(1):68-9.

Sedlak et Lindsay. Anal Biochem. 1968 Oct 24;25(1):192-205. 
$451 / 25$

\section{Polyphenols from Colombian Passiflora Ligularis Juss Regulate Inflammation and Contribute to Weight Control}

Ángel-Isaza, J. ${ }^{1}$; Restrepo-López, JP. ${ }^{1}$; Narváez-Solarte, W. ' ; Carmona-Hernández, JC. ${ }^{2}$; González-Correa, $\mathrm{CH} .^{1}$

${ }^{1}$ Nutrition, Metabolism and Food Safety Research Group. University of Caldas. Colombia; ${ }^{2}$ Medical Reserach Group. University of Manizales. Manizales. Colombia

More than $50 \%$ of adults worldwide suffer from overweightrelated diseases that are connected to chronic low-grade inflammation. The goal in the present work was to evaluate the effect on low-grade inflammation of a Passiflora ligularis Juss (Colombian granadilla) liquid supplementation in overweight mice. Albino mice $(n=36)$ were distributed in 3 treatments (Control, non-supplemented, and supplemented groups) following 12 repetitions. Study groups consumed a high-fat diet and supplementation consisted of $3.0 \mathrm{~g} / \mathrm{L}$ of Passiflora ligularis Juss (granadilla) polyphenol extract. The study lasted 49 days and food and water consumption, inflammatory factors IL- 6 and TNF- $\alpha$, and weight gain were evaluated. Supplementation with Passiflora ligularis Juss (granadilla) extract in mice registered no significant differences in food intake and significant differences in TNF- $\alpha$ levels and weight gain ( $\mathrm{p}<0.05)$. The non-supplemented group yielded a bodyweight increase of $11.5 \%$. The polyphenol-supplemented group registered a weight increase of 5.3\%. Polyphenol from Colombian granadilla $(3.0 \mathrm{~g} / \mathrm{L}$ in the drinking water) shows promising beneficial action against inflammation-related stages and weight gain caused by increased dietary fat. Consumption of polyphenol extracts from granadilla inhibits interleukin-activity and aids in weight control in mice.

Acknowledgments: NUTRIMESA research group.

Financial Support: Universities Caldas and Manizales, and Colciencias.

Conflict of Interest: None.

Authorship: Research question-study design-funding: WNS, CHGC, JCCH, JAA.

Extraction-methodology: JCCH, CHGC.

Bioassay-quantitation: JAI, JPRL.

Manuscript-writing-editing: JAA, JPRL, JCCH, WNS, CHGC.

Keywords: Passifloras-polyphenols-inflammation-weight gain.

\section{References}

Faam B, .... IJBMS. 2014; 17(8): 577-82.

Esser N, ... Diabetes Res Clin Prac. 2014; 105(2):141-150.

Zhang H, Tsao R. Curr Opin Food Sci. 2016; 8:33-42.

Carmona-Hernandez JC, .... ASPR 2017 35(4).

Carmona-Hernandez JC, .... Molecules. 2019 Jan; 24(24):4614.

Torres A. ... ALAN. 2012; 62(4):381-388.

Carmona-Hernandez JC, ... Int J. Food-Sci. 2021; 2021:1-10.

\section{$451 / 26$}

Antioxidant, Anti-Inflammatory, and Immunomodulatory Effects of Soybean Peptide Lunasin in Macrophages and Lymphocytes Models

Hernández-Ledesma, B. '; Paterson Moreno, S. ${ }^{1}$; Fernández-Tomé, S. ${ }^{2}$; Indiano-Romacho, P. ${ }^{1}$

${ }^{1}$ Institute of Food Science Research (CIAL, CSIC-UAM); ${ }^{2}$ Digestive Unit, La Princesa University Hospital, Madrid, Spain

Lunasin is one of the most promising food peptides with positive impact on health ${ }^{1}$. Its antioxidant, anti-inflammatory and immunomodulatory effects were evaluated in $\mathrm{H} 2 \mathrm{O} 2$ - and LPSinduced RAW264.7 macrophages, and mitogen-activated EL-4 lymphocytes. In macrophages, the effects on cell viability and phagocytic capacity, and levels of oxidative-stress and inflammation associated biomarkers were studied. Peptides corresponding to different lunasin's regions with demonstrated bioavailability ${ }^{2}$ were used to elucidate the major contributor on lunasin's effects. Our results showed that lunasin reverted cell viability reducing effects due to $\mathrm{H} 2 \mathrm{O} 2$ and LPS, and provoked a significant reduction of ROS and NO levels in comparison with induced macrophages. These effects were mainly attributed to lunasin's central region although N-terminus peptides also showed activity. Ongoing results in EL-4 lymphocytes will provide further evidence about the effects of lunasin on immune cell proliferation and cytokines secretion.

Acknowledgments: The authors thank the ISCIII by the Sara Borrell fellowship awarded to S. F.-T.

Financial Support: AGL2015-66886-R (MICIU) and PID2019-103919RB-100 (CSIC).

Conflict of Interest: None.

Authorship: S.P., and P.I-R.: experiments; S.F.-T., and B.H.-L.: design and interpretation.

Keywords: Bioactive peptides, lunasin, biomarkers, immunomodulation, cell models.

\section{References}

1 Fernández-Tomé S \& Hernández-Ledesma B (2019) Current state of art after twenty years of the discovery of bioactive peptide lunasin. Food Res Int 116, 71-78.

2 Fernández-Tomé S, Sanchón J, Recio I et al. (2018) Transepithelial transport of lunasin and derived peptides: inhibitory effects on the gastrointestinal cancer viability. J Food Comp Anal 68, 101-110. 
$451 / 28$

Galactooligosaccharide Fibres Exert Immunomodulatory Properties and Interfere with Riboflavin Derivatives in an ex-vivo Study

Del Fabbro, S. ${ }^{1}$; Calder, $P C .{ }^{1,2,3}$; Childs, CE. ${ }^{1,3}$

${ }^{1}$ School of Human Development and Health, Faculty of Medicine, University of Southampton, Southampton, United Kingdom; ${ }^{2}$ NIHR Southampton Biomedical Research Centre, University Hospital Southampton NHS Foundation Trust and University of Southampton, Southampton, United Kingdom; ${ }^{3}$ Institute for Life Sciences, University of Southampton, Southampton, United Kingdom

Mucosal-associated invariant T (MAIT) cells are T lymphocytes with a key role in immune surveillance. Riboflavin derivatives produced by the gut microbiota are MAIT cell ligands ${ }^{(1)}$. Evidence suggests that unknown metabolites synthesised by probiotic strains modulate MAIT cell function ${ }^{(2,3)}$. Galactooligosaccharides (GOS) are prebiotics produced via transgalactosylation by $\beta$-galactosidases, which are expressed by gut bacteria $^{(4)}$. We aim to assess whether GOS modulate the function of MAIT cells or other peripheral blood mononuclear cell (PBMC) subsets and whether cells respond differently to a riboflavin derivative in presence of GOS. Healthy PBMCs $(n=8)$ were cultured for $20 \mathrm{~h}$ with GOS (Bimuno $\left.{ }^{\circledast}\right)(12 \mathrm{mg} / \mathrm{mL})$, or a riboflavin derivative (5-A-RU $0.18 \mu \mathrm{M}+$ methylglyoxal $1 \mu \mathrm{M})$, or co-stimulated with both. Unstimulated cells were used as control. Treatment with GOS did not affect viability. PBMCs incubated with GOS presented lower CD4 expression on T helper cells and secreted more IL- 8 vs control $(p=0.0053$ and $p=0.0005)$. Intracellular staining revealed that monocytes were responsible for the upregulated IL-8 expression. Co-treatment of cells with the riboflavin derivative and GOS resulted in decreased CD69 expression by lymphocytes, T cells and cytotoxic $\mathrm{T}$ cells vs riboflavin derivative alone (all $\mathrm{p}<$ 0.005 ) and in lower \% MAIT cells expressing IL-17A vs riboflavin derivative alone $(\mathrm{p}=0.0008)$. Overall, GOS showed immunomodulatory effects, including the modulation of CD4 expression and enhancement of IL-8 secretion. PBMCs responded differently to ligand challenge in presence of GOS, suggesting that the prebiotic may interfere with riboflavin metabolites or signal via similar pathways.

Acknowledgments: GOS (Bimuno ${ }^{\oplus}$ ) was provided by Clasado Biosciences.

Financial Support: S.D.F. PhD studentship is supported by Clasado Biosciences (grant number 17726/02).

Conflict of Interest: None.

Authorship: S.D.F. carried out the experimental part of the study, analysed the data and drafted the abstract. C. E. C. and P. C. C. supervised and critically revised the study.

Keywords: Galactooligosaccharides, prebiotics, riboflavin derivatives, mucosal-associated invariant T cells, peripheral blood mononuclear cells.

\section{References}

1 Patel O, Kjer-Nielsen L, Le Nours J et al.: Recognition of vitamin B metabolites by mucosal-associated invariant T cells. Nat Commun 2013; 4,1-9.

2 Johansson MA, Bjorkander S, Mata Forsberg M et al.: Probiotic Lactobacilli Modulate Staphylococcus aureus-Induced Activation of Conventional and Unconventional $\mathrm{T}$ cells and NK Cells. Front Immunol 2016;7,1-15.

3 Hinks TS.: Mucosal-associated invariant $\mathrm{T}$ cells in autoimmunity, immune-mediated diseases and airways disease. Immunology 2016;148,1-12.

4 Grimaldi R, Swann JR, Vulevic J et al.: Fermentation properties and potential prebiotic activity of Bimuno ${ }^{\circ}$ galacto-oligosaccharide (65\% galacto-oligosaccharide content) on in vitro gut microbiota parameters. $\mathrm{Br} \mathrm{J}$ Nutr 2016;116,480-486.

\section{$451 / 30$}

\section{The Postbiotic Lactofidus ${ }^{\mathrm{Tm}}$ in Combination with GOS-FOS Prevents Diarrhoea in a Suckling Rat Model of Rotavirus Infection}

\author{
Morales-Ferré, C. ${ }^{1}$; Azagra-Boronat, I. ; Rodríguez-Lagunas, M. ${ }^{1}$; \\ Knipping, $K^{2}{ }^{2}$; Garssen, J. ${ }^{2}$; Pérez-Cano, $F^{1}$ \\ ${ }^{1}$ Physiology Section, Faculty of Pharmacy and Food Science, \\ University of Barcelona; INSA-UB, Spain; ${ }^{2}$ Danone Nutricia \\ Research, Utrecht and Division of Pharmacology Utrecht \\ University, The Netherlands
}

Postbiotics are compounds produced by microorganisms and released from food components or microbial constituents, including non-viable cells that, when administered in adequate amounts, promote health and well-being ${ }^{(1)}$. The aim of this study was to analyse the effects of a postbiotic in combination with prebiotics on rotavirus (RV) infection in suckling rats.

Suckling Lewis rats received daily the vehicle (REF) or the combination of the prebiotic mixture (galacto-oligosaccharides and fructo-oligosaccharides, GOS-FOS) and the postbiotic (LactofidusTM), including the human milk oligosaccharide (HMO) 3'- galactosyllactose (GL), and on day 5 also the RV. Faecal samples were clinically evaluated, and immune and microbial variables were analysed.

The prebiotic and postbiotic mixture administration reduced the severity of diarrhoea up to $\sim 50 \%$ and the incidence around $\sim 60 \%(\mathrm{p}<0.05)$. Viral shedding and intestinal permeability were also attenuated. The supplementation showed a dose dependent blocking activity. Although no changes in level of anti-RV antibodies were observed some changes in the inmunoglobulin profile was observed. Changes in gut microbiota were also accompanied by changes in short chain fatty acids production. An enhancement of innate defences, bacterial interaction and intestinal maturation was induced as shown by the gene expression up-regulation of mucin 3 and, some Toll-like receptors and the down-regulation of the immaturity marker FcRn.

In conclusion, the combination of postbiotics, including $\mathrm{HMO}$ 3'-GL (Lactofidus ${ }^{\mathrm{TM}}$ ) with GOS-FOS mixture supplementation during suckling reduces the severity of diarrhoea caused by the RV and 
induces clinical amelioration by direct immune and microbiome effects, promoting a more effective defensive response.

Acknowledgments: We would like to acknowledge the Centre of Scientific Services of the University of Barcelona (Genomics and Chromatography Units and the Animal facility at the Faculty of Pharmacy).

Financial Support: This project has been funded by Danone Nutricia Research (Project FBG 309956).

Conflict of Interest: Karen Knipping and Johan Garssen are employees of Danone Nutricia Research.

Authorship: KK, JG, MR-L and FP-C designed the study. IA-B, C-M-F, MJR-L and FP-C were involved in the experiments; all of them interpreted the results and drafted the abstract.

Keywords: Rotavirus, postbiotics, prebiotics, diarrhoea, microbiota.

\section{Reference}

1 Collado, M.C.; Vinderola, G.; Salminen, S. Postbiotics: Facts and open questions. A position paper on the need for a consensus definition. Benef. Microbes 2019, 10, 711-719.2. Malagón-rojas, J.N., Mantziari, A., Salminen,S.,\& Szajewska,H.Postbiotics for preventing and treating common infectious diseases in children: A systematic review. Nutrients. 2020;12(2). https://doi.org/10.3390/nu12020389

\section{$451 / 31$ \\ Influence of a Postbiotic Dietary Intervention on the Immune System Development}

Morales-Ferré, C. '; Massot-Cladera, M. ${ }^{1}$; Rodríguez-Lagunas, M. ${ }^{1}$; Knipping, K. ${ }^{2}$; Garssen, J. ${ }^{2}$; Pérez-Cano, F. ${ }^{1}$

${ }^{1}$ Physiology Section, Faculty of Pharmacy and Food Science, University of Barcelona and INSA-UB, Spain; ${ }^{2}$ Danone Nutricia Research, Utrecht and Division of Pharmacology Utrecht University, The Netherlands

Breast milk is the best source of nutrition for infants including bioactive compounds that influence brain, gastrointestinal tract and immune system development. Postbiotics are functional bioactive microbial-derived compounds inspired by the presence of (non-)viable bacteria and their metabolites in breast milk and may be used to promote the infant health ${ }^{(1)}$. Thus, the aim of this study was to analyse the overall impact on gut and immune markers of a particular postbiotic in suckling rats.

Forty neonatal suckling Lewis rats were daily supplemented with postbiotics, including the human milk oligosaccharide (HMO) 3'- galactosyllactose (GL) (LactofidusTM) by oral gavage. Different variables such as the body and organ weights, faecal data, stomach content $\mathrm{pH}$, haematology variables, immunoglobulin profile and intestinal gene expression were measured. Caecal microbiota composition and short chain fatty acids (SCFA) production were analysed and Spearman correlation coefficient was used to search for correlation between both variables.

The dietary intervention with the postbiotic did not affect the animals' growth, the haematological variables or the organs' weight, with exception of the small intestine which showed an increase on day 8 in the POST animals. In addition, no differences were observed on faecal weight or appearance. A slight modulation of the immunoglobulin profile and microbiota composition were observed without an effect on SCFA production. However, a clear up-regulation of different Toll like receptors gene expression was observed.

In conclusion, the postbiotic studied may have an impact on the maturation of the developing microbiota and immune system of the infant.

Acknowledgments: We would like to acknowledge the Centre of Scientific Services of the University of Barcelona (Genomics and Chromatography Units and the Animal facility at the Faculty of Pharmacy).

Financial Support: This project has been funded by Danone Nutricia Research (Project FBG 309956).

Conflict of Interest: Kees van Limpt, Karen Knipping and Johan Garssen are employees of Danone Nutricia Research.

Authorship: KK, JG, MR-L and FP-C designed the study. MM, CM-F, MJR-L and FP-C were involved in the experiments; all of them interpreted the results and drafted the abstract.

Keywords: Microbiota, postbiotics, suckling rats, immunoglobulins, immune system.

\section{Reference}

1 Collado, M.C.; Vinderola, G.; Salminen, S. Postbiotics: Facts and open questions. A position paper on the need for a consensus definition. Benef. Microbes 2019, 10, 711-719.

\section{$451 / 32$ \\ Effect of High Salt Diet on Vascular Reactive Oxygen Species Production in Tff3-/-/C57BL/6N Knockout Mice}

Kozina, N.; Jukic, I.; Drenjancevic, I.

Faculty of Medicine Osijek, University Josip Juraj

Strossmayer Osijek, Croatia

Introduction: Tff3 gene knockout mice (Tff3-/-/C57BL/6N) have changes in lipid metabolism which may affect vascular function $^{(1,2)}$. This study aimed to asses the effect of high salt (HS) diet on endothelial reactive oxygen species (ROS) production in carotid arteries of Tff3-/- knockout mice and their wild type controls (WT, C57BL/6N).

Methods: Male, ten-weeks-old transgenic Tff3-/-/C57BL/6N and $\mathrm{WT} / \mathrm{C} 57 \mathrm{BL} / 6 \mathrm{~N}$ (parental strain) healthy mice were divided in LS $(0.4 \% \mathrm{NaCl})$ and $\mathrm{HS}(4 \% \mathrm{NaCl}$ in rodent chow fed for 1 week) groups. After anaesthesia (ketamine-chloride and midazolam), mice were decapitated and carotid arteries were isolated and cannulated on pressure myograph with $(\Delta 80 \mathrm{mmHg})$ or without flow $(\Delta 0 \mathrm{mmHg})$, in the absence/presence of the superoxide dismutase mimetic TEMPOL $(100 \mu \mathrm{mol} 1-1)$. ROS production was determined by dihydroethidine (DHE, $20 \mu \mathrm{M}$ ) fluorescence assay. 
Statistical analyses were performed with One-way ANOVA test; $\mathrm{p}<0.05$ was considered significant. All experimental procedures conformed to the European Guidelines for the Care and Use of Laboratory Animals (directive 86/609) and were approved by local and national Ethical Committee (No.2158/61-02-139/2-06; No.2158/61-07-14-119).

Results: Basal (no-flow) ROS production was significantly increased in $\mathrm{WT}_{-}$HS compared to the $\mathrm{WT}_{-}$LS group and in Tff3-/__HS compared to Tff3-/__LS group.

Tempol significantly decreased ROS production in $\mathrm{WT}_{-} \mathrm{HS}$ and Tff3-/-_HS groups.

Under flow conditions, ROS production was significantly increased in WT_HS compared to WT_LS group and in Tff3-/-_ HS compared to Tff3-/-_LS group. Tempol significantly decreased ROS production in all groups.

Conclusion: HS diet increases generation of ROS and increases vascular oxidative stress in both strains.

Acknowledgments: The author would like to thank all the members from the group Department of Physiology and Immunology, Faculty of Medicine Osijek.

Financial Support: This study was supported by the Croatian Science Foundation under the project IP-2014-09-6380 (V-ELI Athero), VIF-2018-MEFOS-09-1509 grant and Faculty of Medicine Osijek Institutional grant \#IP-1-MEFOS2019 and \#IP-2MEFOS2020 (PI Ines Drenjančević).

Conflict of Interest: None.

Authorship: N. Kozina: conceived and designed the research, performed the experiments, analysed the data, interpreted the results of the experiments, prepared the figures, writing the abstract, approved the final version of the abstract.

I. Drenjancevic: conceived and designed the research, analysed the data, interpreted the results of the experiments, writing the abstract, approved the final version of the abstract.

I. Jukić: conceived and designed the research, analysed the data, interpreted the results of the experiments, writing the abstract, approved the final version of the abstract.

Keywords: Tff3 gene, high salt diet, reactive oxygen species, fluorescence.

\section{References}

1 Bujak, M.; Tartaro Bujak, I.; Sobocanec, S.; Mihalj, M.; Novak, S.; Cosic, A.; Tolusic Levak, M.; Kopacin, V.; Mihaljevic, B.; Balog, T.; et al. Trefoil Factor 3 DeficiencyAffects Liver Lipid Metabolism. Cell. Physiol. Biochem. 2018, 47, 827-841. [CrossRef] [PubMed].

2 Kozina, N.; Jukic, I. Trefoil Factor Family (TFF): Peptides with Numerous Functions. SEEMEDJ 2019, 3, 69-77 [CrossRef].

\section{$451 / 33$ \\ Vitamin and Mineral Deficiency may Impair Immunity: Dietary Intake Analysis in Galician (Spain) Population}

Sinisterra-Loaiza, LI. ${ }^{1}$; Alonso-Lovera, P. $^{2}$; Cardelle-Cobas, A. ${ }^{2}$; Vázquez Belda, B. ${ }^{2}$; Cepeda Sáez, A. $^{2}$

${ }^{1}$ Laboratorio de Higiene Inspección y Control de Alimentos, Universidad de Santiago de Compostela; ${ }^{2}$ LHICA- Depto. Química Analítica, Nutrición y Bromatología, Universidade de Santiago de Compostela

The objective of this study was to evaluate the intake of specific vitamins and minerals and its adequacy to the Recommended Dietary Allowance (RDA) in a sample of Galician population. It is well known that some vitamins and minerals are necessary for the correct functioning of the immune system ${ }^{1}$. Certain populations have shown an inadequate dietary intake (DI) and considering that situations with increased requirements (e.g., infections and stress) further decrease stores within the body, the identification of lower intakes through diet in the population could be of great interest since its correction would contribute to reduce the risk and severity of infections as in the case of infection by Covid- 19 .

In this study, two-hundred volunteers were asked to fill a 3-day food intake record ( 2 weekdays and 1weekend day). The mean of the three dietary recalls was used to estimate DI and their adequacy based on the RDA for Spanish population ${ }^{2}$. The obtained results showed a deficiency in vitamin D intake, as well as in vitamin A, for more than half of the volunteers whereas for other vitamins as vitamin $\mathrm{C}$, the RDA was reached in all cases. Among the minerals, is noteworthy the case of Fe for which the RDA was non-achieved in women.

The analysis of specific micronutrients intake in a sample of Galician population was carried out by the first time showing an important deficiency for some of them. This deficiency if prolonged in time, could negatively impact on their immune system, make it more susceptible to infections.

Acknowledgments: The authors thank the CyTED for funding the IBEROBDIA project (918PTE0540) and the Ministry of Economy and Competitiveness for the financial support for this project through the State Program of $\mathrm{I}+\mathrm{D}+\mathrm{I}$ Oriented to the Challenges of Society 2017-2020 (International Joint Programming 2018). Projects PCI2018-093245.

Financial Support: The authors thank the CyTED for funding the IBEROBDIA project (918PTE0540) and the Ministry of Economy and Competitiveness for the financial support for this project through the State Program of $\mathrm{I}+\mathrm{D}+\mathrm{I}$ Oriented to the Challenges of Society 2017-2020 (International Joint Programming 2018). Projects PCI2018-093245.

Conflict of Interest: None.

Authorship: Laura Isabel Sinisterra-Loaiza and Patricia Alonso lovera carried out the work with the volunteers (captation, informed consent and 72 h-questionnaire), Beatriz Vazquez Belda and Alejandra Cardelle-Cobas supervised the work (thow fill the questionaries together with the volunteeers, data obtaining, etc) and professor Alberto Cepeda designed the work as the PI of the project in which this work is included. 
Keywords: Vitamins, minerals, immune system, Galician, dietary intake.

\section{References}

Gombardt, A. F., Pierre, A., Maggini, S. (2020). A review of Micronutrients and the Inmune System-working in harmony to reduce the risk of infection. Nutrients, $12,236$.

FESNAD (2010). Ingestas dietéticas de Referencia (IDR) para la población española, 2010. Actividad Dietética, 14(4):196-197.

\section{$451 / 34$}

\section{N-3-Polyunsaturated Fatty Acids-Enriched Eggs Consumption Increases Serum Concentrations of E-Series Resolvins in Young Healthy Participants}

Kolobarić, N.; Matić, A.; Šušnjara, P.; Mihalj, M.; Drenjancevic, I.

Faculty of Medicine, Josip Juraj Strossmayer University of Osijek, Croatia

Introduction: Western diet has lately been characterized by increased intake of n-6 PUFAs and decline in n-3 PUFAs intake1, 2 which leads to disbalance between pro- and anti-inflammatory lipid mediators synthesized from fatty acid precursors and consequently higher risk for chronic disease development3, 4 .

Materials and Methods: Forty young healthy volunteers of both sexes, 19-28 years old, were divided into: Control group (consumed regular hen eggs) and n-3 PUFA group (consumed enriched hen eggs containing additional $\alpha$-linolenic (ALA), eicosapentaenoic (EPA) and docosahexaenoic acid (DHA) in edible part of hard-boiled egg). Venous blood samples were collected before and after three-weeks dietary protocol. Serum concentrations of leukotrienes (B4, B5), prostaglandins (E2, E3) and resolvin (RvE1) were measured by commercially available ELISA kits.

Results: Serum concentrations of LTB5 was increased in both Control and n-3 PUFA groups after the protocol, compared to each baseline $(\mathrm{p}<0.0001$ and $\mathrm{p}=0.012$, respectively). RvE1 endpoint serum concentration significantly increased $(p=0.013)$, while prostaglandin E2/E3 serum concentrations ratio significantly decreased $(\mathrm{p}=0.014)$ in $\mathrm{n}-3$ PUFA group compared to baseline.

Discussion: Our results confirm previously elaborated inhibitory effect of supplemental n-3 PUFAs on COX pathway metabolites in various cell lines5, 6, although decreased PGE2/PGE3 ratio suggests somewhat anti-inflammatory effect. Therefore, increased pro-resolving mediator RvE1 production indicates that protective effect of n-3 PUFAs in healthy individuals is mainly exhibited through enzymatic pathways producing these metabolites.

Conclusion: Anti-inflammatory effect of n-3 PUFA functional foods is manifested through higher production of pro-resolving mediators, rather than minimally inflammatory eicosanoids, in healthy population.

Acknowledgments: Special thanks to all the participants for their valuable contribution to the study.
Financial Support: Study was funded by European Structural and Investment Funds, to Science Centre of Excellence for Personalized Health Care, Josip Juraj Strossmayer University of Osijek, Scientific Unit for Research, Production and Medical Testing of Functional Food, \# KK.01.1.1.01.0010.

Conflict of Interest: None.

Authorship: Conceptualization, N.K., A.M. and I.D.; methodology, N.K., P.Š., A.M., M.M. and I.D.; software, N.K., A.M. and M.M.; validation, N.K., M.M., I.D.; formal analysis, N.K., M.M. and A.M.; investigation, N.K., P.S. and A.M.; resources, I.D.; writing, N.K. and A.M.; M.M., P.Š., A.M. and I.D.; visualization, N.K., A.M. and I.D.; supervision, I.D.; project administration, N.K., M.M. and I.D.; funding acquisition, I.D.

Keywords: N-3 PUFA, functional food, eicosanoids, resolvins, inflammation.

\section{References}

1 Wijendran V, Hayes KC. Dietary n-6 and n-3 fatty acid balance and cardiovascular health. Annu Rev Nutr. 2004; 24:597-615.

2 Yang LG, Song ZX, Yin H, Wang YY, Shu GF, Lu HX, et al. Low n-6/n-3 PUFA Ratio Improves Lipid Metabolism, Inflammation, Oxidative Stress and Endothelial Function in Rats Using Plant Oils as n-3 Fatty Acid Source. Lipids. 2016 Jan;51(1):49-59.

3 Husted KS, Bouzinova EV. The importance of n-6/n-3 fatty acids ratio in the major depressive disorder. Medicina. 2016 Jan 1;52(3):139-47.

4 Nindrea RD, Aryandono T, Lazuardi L, Dwiprahasto I. Association of Dietary Intake Ratio of n-3/n-6 Polyunsaturated Fatty Acids with Breast Cancer Risk in Western and Asian Countries: A Meta-Analysis. Asian Pac J Cancer Prev. 2019;20(5):1321-7.

5 Araujo P, Belghit I, Aarsæther N, Espe M, Lucena E, Holen E. The Effect of Omega-3 and Omega-6 Polyunsaturated Fatty Acids on the Production of Cyclooxygenase and Lipoxygenase Metabolites by Human Umbilical Vein Endothelial Cells. Nutrients. 2019 Apr 27;11(5):966.

6 Benistant C, Achard F, Marcelon G, Lagarde M. Platelet inhibitory functions of aortic endothelial cells. Effects of eicosapentaenoic and docosahexaenoic acids. Atherosclerosis. 1993 Dec;104(1-2):27-35.

\section{$451 / 35$}

\section{Changes in the Adipokine Profile of Human Milk throughout Lactation Due to Premature Delivery}

Grases-Pintó, B.; Rodríguez-Lagunas, M.; Castell Escuer, M.; Pérez-Cano, F.; Franch Masferrer, Á.

Faculty of Pharmacy and Food Science, UB; Nutrition and Food Safety Research Institute (INSA.UB)

In preterm newborns the immaturity of the immune system is remarkable. Breastfeeding has a key role promoting the maturation of newborn's immune system due to milk's rich composition in bioactive compounds ${ }^{(1)}$. This study aimed to analyze the changes in human milk adiponectin, leptin and resistin concentration corresponding to colostrum, transitional and mature milk from mothers delivering at term $(\mathrm{T})$, preterm $(\mathrm{PT})$ and very preterm (VPT). Concentrations of these adipokines were quantified in milk samples by a multiplex bead-immunoassay technique. Adiponectin concentration was lower on colostrum from PT group compared 
to T group. Conversely, all three types of milk from VPT group had the highest concentration of this adipokine. Concerning leptin content, milk from PT group contained higher concentration of this adipokine at the three time points of lactation than that in the $\mathrm{T}$ group. Moreover, in the three groups of study, resistin had the highest concentration in colostrum with respect to transitional or mature milk. Moreover, compared to PT group, the colostrum from the VPT group had lower resistin concentration. Considering all groups, a positive correlation between transitional and mature milk was detected for the three adipokines. Taking together all the samples throughout the lactation period, although a negative correlation was observed between adiponectin and resisitin, a positive link was identified between leptin and resistin from PT and VPT considered together. Globally, these results demonstrate the effect of gestational age on the adipokine profile of human milk.

Acknowledgments: The authors would like to thank Lidia Marín-Morote for their help with the laboratory work.

Financial Support: This research was funded by the Spanish Ministry of Economy, Industry and Competitiveness (AGL201348459-P). B.G.-P. holds a fellowship from the Spanish Ministry of Economy, Industry and Competitiveness (BES-2014-068134).

Conflict of Interest: The authors declare no competing interests.

Authorship: The authors' contributions were as follows: À.F., M.C. and F.J.P.-C. conceived and designed the research; all authors carried out the experiments and the data analysis and were involved in the interpretation of the data; B.G.-P., F.J.P.-C. and À.F. contributed to the initial draft of the abstract. All authors have read and approved the final version of the abstract.

Keywords: Breast milk, prematurity, adiponectin, leptin, resistin.

\section{Reference}

1 Gila-Diaz, A.; Arribas, S.M.; Algara, A.; Martín-Cabrejas, M.A.; López de Pablo, L. Á.; Sáenz de Pipaón, M.; Ramiro-Cortijo, D. A review of bioactive factors in human breastmilk: a focus on prematurity. Nutrients (2019) 11, 1307.

\section{$451 / 36$ \\ Four Nutrients - Enriched Hen Eggs Consumption Improves Skin Microvascular Reactivity in Healthy Individuals}

Šušnjara, P.; Stupin, A.; Kolobarić, N.; Mihaljević, Z.; Drenjancevic, I.

Institute and Department of Physiology and Immunology, Faculty of Medicine Osijek, Croatia

Introduction: In addition to basic nutrients necessary for the growth and development of the organism, functional food also contains ingredients that improve health or reduce the risk of developing chronic diseases ${ }^{1}$. Recently we demonstrated that consumption of hen eggs enriched with n-3 polyunsaturated fatty acids (n-3 PUFAs) improves microvascular blood flow ${ }^{2,3}$. The aim of this study was to determine the effect of four nutrients - enriched hen eggs (n-3 PUFA, lutein, vitamin $\mathrm{E}$ and selenium) on microvascular reactivity in healthy young subjects ${ }^{4}$.

Material and Methods: Healthy young subjects of both sexes were divided into two groups, control group $(\mathrm{N}=9$, consumed 3 regular eggs) and Quatro group $(\mathrm{N}=14$, consumed 3 enriched eggs) in morning, daily, for three weeks. Endothelium-dependent vasodilation to iontophoretically administered acetylcholine (ACh) and to post-occlusive reactive hyperemia (PORH) and iontoforetically administered sodium-nitroprusside ((SNP); measure of endothelium-independent vasodilatation) were assessed by Laser Doppler Flowmetry. Vascular reactivity in microcirculation was assessed on the first and last day of dietary protocol1.

Results: Vasodilation in response to ACh and PORH was significantly enhanced after dietary protocol within Quatro group. Response to SNP remained unchanged in both groups.

Conclusions: Consumption of hen eggs enriched with 4 nutrients improved microcirculatory blood flow in response to acetylcholine and reactive hyperemia in young healthy subjects. Since serum concentration of n-3 PUFAs was significantly higher in the group of subjects who consumed eggs enriched with four nutrients, results suggest beneficial effects of functional food consumption in microcirculation.

Acknowledgments: The author would like to thank all the members from the group Department of Physiology and Immunology, Faculty of Medicine Osijek.

Financial Support: The research is funded by the Croatian National Science Center of Excellence for Personalized Health Care,JosipJurajStrossmayer UniversityOsijek\# KK.01.1.1.01.0010.

\section{Conflict of Interest: None.}

Authorship: Conceptualization, P.Š., A.S. and I.D.; methodology, P.Š., A.S., N.K., Z.M., and I.D.; software, P.Š., A.S. and I.D.,; validation, P.Š., A.S., I.D.; formal analysis, P.Š., A.S. and I.D.; investigation, P.Š. N.K., Z.M. and A.S.; resources, I.D.; writing, P.Š., and N.K.; Z.M., A.S. and I.D.; visualization, P.S., A.S. and I.D.; supervision, I.D.; project administration, P.Š., A.S. and I.D.; funding acquisition, I.D.

Keywords: Endothelium dependent and independent vasodilation, functional food, microvascular reactivity, n-3 PUFAs.

\section{References}

1 Stupin, Ana; Mihalj, Martina; Kolobarić, Nikolina; Šušnjara, Petar; Kolar, Luka; Mihaljević, Zrinka; Matić, Anita; Stupin, Marko; Jukić, Ivana; Kralik, Zlata et al. Anti-Inflammatory Potential of n-3 Polyunsaturated Fatty Acids Enriched Hen Eggs Consumption in Improving Microvascular Endothelial Function of Healthy Individuals- Clinical Trial// International journal of molecular sciences, 21 (2020), 11; 4149, 20 doi:. org/10.3390/ijms21114149.

2 Stupin, Marko; Kibel, Aleksandar; Stupin, Ana; Selthofer-Relatić, Kristina; Matić, Anita; Mihalj, Martina; Mihaljević, Zrinka; Jukić, Ivana; Drenjančević, Ines, The physiological effect of $\mathrm{n}-3$ polyunsaturated fatty acids (n-3 PUFAs) intake and exercise on hemorheology, microvascular function and physical performance in health and cardiovascular diseases; is there an interaction of exercise and dietary n-3 PUFA intake? // Frontiers in Physiology, 10 (2019), 1129, 19 doi:10.3389/fphys.2019.01129.

3 Kolobarić, N., Gradinjan Centner, M., Šušnjara, P., Matić, A. \& Drenjančević, I. (2020) Anthropometric and Biochemical Parameters in Relation to Dietary Habits as Early Indicator of Cardiovascular Impairment in Young Adult Cohort. International journal of environmental research and public health, 17 (24), 1-14 doi:10.3390/ijerph17249208. 
4 Galović, Olivera; Nikolić, Marija Elena; Dornjak, Luka; Marunica, Matea; Kralik, Zlata Sample preparation and analysis of nutricines content in table eggs // Book of Abstract of 18th Ružička days TODAY SCIENCE - TOMORROW INDUSTRY, Vukovar, Croatia, 2020. str. 30-30.

\section{$451 / 37$ \\ Dietary Supplementation with Spray-Dried Animal Plasma Presents Neuroprotective Effects in Senescence Mice}

Rosell Cardona, C1; Miró, L. ${ }^{1,2}$; Polo, J. ${ }^{2}$; Amat, C. ${ }^{1}$; Moretó, M. ${ }^{1}$; Pérez-Bosque, $A{ }^{1}$

${ }^{1}$ Department of Biochemistry and Physiology, Faculty of Pharmacy and Food Science, UB; ${ }^{2}$ APC-Europe, SLU, Spain

Dietary supplementation with spray-dried animal plasma (SDP) has anti-inflammatory effects in different models of inflammation [1-3]. This research aimed to know if dietary supplementation with SDP could prevent the expression of markers of neuroinflammation related to Alzheimer's disease (AD), as those involved in microgliosis and astrogliosis. Experiments were performed on SAMP8 male mice. The groups were: 2 months-old (reference young, 2M) and 6 months-old (senescent) mice fed either a Control diet (6M-CTL) or a diet containing 8\% SDP (6M$\mathrm{SDP}$ ) for 4 months. Glial markers and cytokine expression were analyzed in brain tissue by real-time PCR, while AD markers (p-Tau, p-GSK3beta and p-CDK5) were determined by Western blot. Senescent mice had increased the expression of microgliosis markers, such as Trem 2 and Arg1 $(\mathrm{p}<0.05)$, as well as the marker of astrogliosis GFAP $(\mathrm{p}<0.05)$, and all of them were reduced by SDP supplementation (all, $\mathrm{p}<0.05)$. Senescent mice presented higher abundance of $\mathrm{p}$-Tau and $\mathrm{p}-\mathrm{CDK} 5$ proteins and lower abundance of p-GSK3beta protein. Dietary supplementation with SDP also prevented $\mathrm{AD}$ hallmarks alterations in aged mice (all, $\mathrm{p}<0.05$ ). Moreover, the expression of pro-inflammatory cytokines, such as IL-18 and IL-6, was augmented in senescent mice brain (both, $\mathrm{p}<0.05)$, and was attenuated by SDP supplementation $(\mathrm{p}<0.05)$. On the other hand, senescent mice fed SDP diet presented more Tgfbeta in brain tissue $(\mathrm{p}<0.05)$. These results indicate that dietary supplementation with SDP attenuates neuroinflammation by reducing astrogliosis and microgliosis and delays the onset of $\mathrm{AD}$ in aged SAMP8 mice.

Acknowledgments: We are grateful to M. Pallàs and C. Griñán for giving antibodies of AD markers.

Financial Support: This study was funded by APC-Europe SLU (Granollers, Spain) by research contracts with the Bosch i Gimpera Foundation of the University of Barcelona. The research group was also supported by grant 2017SGR945 for Consolidated Research Groups, Generalitat de Catalunya, Spain. CRC was supported by a grant from Bosch i Gimpera Foundation; APC Europe S.L.U. (Granollers, Spain) provided the experimental diets.

Conflict of Interest: AP-B, CA, CRC, MM have no conflicts of interests. JP and LM are employed by APC-Europe SLU. The funding sponsors had no role in the design of the study; in the collection, analysis, or interpretation of data; in the writing of the abstract, and in the decision to publish the results.
Authorship: AP-B, CA, JP and MM conceived and designed the experiments; AP-B, LM and CRC performed the experiments; AP-B, LM, CRC and MM analyzed the data; AP-B, LM and CRC wrote the abstract. JP contributed with essential reagents and materials. All authors discussed the results and edited the communication. All authors read and approved the final communication.

Keywords: Neuroinflammation, dietary supplementation, Alzheimer disease, plasma proteins.

\section{References}

1 Garcia-Just A, et al. J Nutr. 2020; 150(2):303-311. doi: 10.1093/jn/ nxz239.

2 Miró L, et al. Int J Mol Sci. 2020; 21(18):6760. doi: 10.3390/ijms21186760.

3 Pérez-Bosque A, et al. Nutrients. 2016; 8(10):657. doi: 10.3390/ nu8100657.

\section{$451 / 38$}

\section{Dietary Plasma Protein Supplementation} Ameliorates Intestinal Infection by Escherichia Coli in Young Mice

Miró, L. ${ }^{1,2}$; Rosell Cardona, C. ; Polo, J. ${ }^{2}$; Amat, C. ${ }^{2}$; Moretó, M. ${ }^{1}$; Pérez-Bosque, $A .^{2}$

${ }^{1}$ Department of Biochemistry and Physiology, Faculty of Pharmacy and Food Science, UB; ${ }^{2}$ APC-Europe, S.L.U, Spain

Previous experiments have shown that dietary supplementation with spray-dried animal plasma (SDP) attenuates toxin induced intestinal and lung inflammation (1-3). The aim of this study was to assess whether supplementation with SDP can reduce the magnitude of intestinal infection induced by oral administration of Escherichia coli O42 (EC). Male C57BL/6 mice were fed with Control diet or with a diet supplemented with 8\% SDP for 14 days. After 9 days on diet, mice received an intragastric challenge of $10^{8} \mathrm{CFU}$ of EC. After 5 days post infection (dpi), leukocyte recruitment and activation were analysed in mesenteric lymph nodes (MLN), and cytokine expression was determined in lymphocytes from MLN and colon mucosa by real time PCR. The EC administration increased the leukocyte recruitment in MLN as well as the percentage of activated Th lymphocytes $(\mathrm{P}<0.05)$. SDP supplementation reduced both the recruitment of lymphocytes as well as their activation $(\mathrm{P}<0.05)$. EC administration increased the expression of IL- 6 and IL-1 $\beta$ in lymphocytes of MLN and colon mucosa (all $\mathrm{P}<0.05$ ). Dietary supplementation with SDP attenuated the pro-inflammatory cytokine expression in both tissues (all $\mathrm{P}<0.05)$. On the other hand, SDP supplementation increased the expression of Foxp 3 in colon tissue $(\mathrm{P}<0.05)$. Moreover, SDP supplemented mice with EC infection showed a reduced abundance of pathogenic EC in feces 5 dpi. Supplementation with animal plasma proteins prevents the activation of the immune system induced by EC infection.

Acknowledgments: We are grateful to A. Juárez and A. Prieto for bacteria production. 
Financial Support: This study was funded by APC-Europe SLU (Granollers, Spain) by research contracts with the Bosch i Gimpera Foundation of the University of Barcelona. The research group was also supported by grant 2017SGR945 for Consolidated Research Groups, Generalitat de Catalunya, Spain. CRC was supported by a grant from Bosch i Gimpera Foundation; APC Europe S.L.U. (Granollers, Spain) provided the experimental diets.

Conflict of Interest: AP-B, CA, CRC, MM have no conflicts of interests. JP and LM are employed by APC-Europe SLU. The funding sponsors had no role in the design of the study; in the collection, analysis, or interpretation of data; in the writing of the manuscript, and in the decision to publish the results.

Authorship: AP-B, CA, JP and MM conceived and designed the experiments; AP-B, LM and CRC performed the experiments; AP-B, LM, CRC and MM analyzed the data; AP-B, LM and CRC wrote the abstract. JP contributed with essential reagents and materials. All authors discussed the results and edited the communication. All authors read and approved the final communication.

Keywords: Intestinal infection, dietary supplementation, SDP, plasma proteins, nutrition.

\section{References}

1 Garcia-Just A, et al. J Nutr. 2020; 150(2): 303-311. doi: 10.1093/jn/ nxz239.

2 Miró L, et al. Int J Mol Sci. 2020; 21(18): 6760. doi: 10.3390/ijms21186760.

3 Pérez-Bosque A, et al. Nutrients. 2016; 8(10):657. doi: 10.3390/ nu8100657.

$451 / 39$

Immunonutritional agonists from S. hispanica $L$ Improve Innate Immune Control of the Alterations at Central Nervous System during Hepatocarcinogenesis

Laparra Llopis, J. ${ }^{1}$; Garcia Tejedor, $A .^{2}$

${ }^{1}$ Madrid Institute for Advanced Studies in Food (Imdea Food);

${ }^{2}$ Valencian International University

This study aimed to evaluate the role of immunonutritional protease inhibitors (ImPIs) from Salvia hispanica L to influence the neuro-immune activity in a preclinical hepatocarcinoma (HCC)-developing mice model fed a high fat diet (HFD). ImPIs exerted a significant influence on energy expenditure and food intake. Feeding ImPIs enabled to counteract the alterations in the brain-associated macrophages' M1/M2 phenotypes showing normalized proportions of those in relation to healthy animals. Animals fed with ImPIs displayed a better controlled production of inflammatory mediators as GM-CSF an essential cytokine to develop encephalomyelitis. Accordingly, there was a trend decreasing brain edema in animals administered with ImPIs. These effects were associated to positive changes in the production of neurotransmitters implied in both brain activation and NRLP3 inflammasome-dependent neuroinflammation. Thus, it is concluded that ImPIs from S. hispanica $\mathrm{L}$ activate the gut-brain axis improving immune control of neuroinflammation caused during HCC development.

Acknowledgments: JML thanks MICINN for his 'Ramon y Cajal' contract (RYC-2015-18083). Authors thank staff from the animal facility at CIB-CSIC for their technical assistance in animal experiments.

Financial Support: This work was financially supported by grant Food4ImNut [PID2019-107650RB-C22, Ministry of Science, Innovation and Universities - MICIU].

Conflict of Interest: The authors declare that they have no conflict of interest.

Authorship: Both authors have contributed equally to formal analysis, data curation, managing resources and writing and revising the document.

Keywords: Neuroimmune axis, serine-type protease inhibitors, Salvia hispanica L, macrophages.

\section{References}

N/A

\section{$451 / 40$ \\ Glycaemic Control, Cytokines and Vitamin D in Adults with Type 2 Diabetes Mellitus in Mexico \\ Valdés-Ramos, R. ${ }^{1}$; Román Casas, M. '; Martínez-Carrillo, B. ${ }^{1}$; Guadarrama-López, A. ${ }^{2}$ \\ ${ }^{1}$ Faculty of Medicine. Autonomous University of the State of México; ${ }^{2}$ Multidisciplinary Health Clinic and Faculty of Medicine. Autonomous University of the State of México}

Aim: To correlate glucose, insulin, cytokines, adipokines and vitamin $\mathrm{D}$ in adults with type 2 diabetes mellitus in Mexico (T2DM).

Background: Diabetes mellitus is a chonic and multifactorial disease that affects a lot of people around the world; according to the WHO there are 422 millon people with diabetes, $90 \%$ of whom have T2DM (1). Currently, scientific evidence suggests that patients with T2DM have low concentrations of vitamin D and systemic inflammation; these factors aggravate symptoms and cause complications (2-4).

Study design: A descriptive observational study.

Materials and Methods: This study is a secondary analysis of a database, which included 93 adults with T2DM selected from outpatient clinics from Toluca, State of Mexico. The database included glucose, insulin, Hb1Ac, cytokines, adipokines and vitamin D status; these data were selected and statistically analyzed.

Results: A significative correlation $(\mathrm{P}<0.05)$ was found between IL-1B, IL-10, IL-8, IL-6 IL-12, TNFa and IFN- $\gamma$ with vitamin $\mathrm{D}$ serum concentrations. Leptin had a positive correlation with insulin and HOMA-B. Also, glucose correlated with $\mathrm{Hb} 1 \mathrm{Ac}$, leptin, IL-12, IFN- $\gamma$, insulin, HOMA-B and HOMA-IR. Insulin was associated with HOMA-B and HOMA- IR. Finally, a significant deficiency of vitamin $\mathrm{D}$ in all patients was found $(<20 \mathrm{ng} / \mathrm{ml})$. 
No significant changes were found between vitamin D transporting protein and other markers.

Conclusion: Mexican population with T2DM had a significant deficiency of vitamin $\mathrm{D}$ which associated with cytokine markers. Additionally, vitamin D deficiency aggravates T2DM symptoms and development of complications.

Acknowledgments: The authors thank the Universidad Autónoma del Estado de México (UAEMex) and the Consejo Nacional de Ciencia y Tecnología (CONACYT).

Financial Support: The project from which this abstract is derived was funded by the Consejo Nacional de Ciencia y Tecnología (CONACyT) and is part of MRC's Doctoral Thesis.

Conflict of Interest: All authors declare that they have no conflict of interest.

Authorship: RV-R: Participated with data collection and analysis.

MRC: Participated with data collection and analysis.

BM-C: Participated with sample collection and biochemical measurements.

ALGL: Participated with sample collection and biochemical measurements.

All authors participated in the writing and revision of the abstract.

Keywords: Type 2 diabetes mellitus, glycaemic control, cytokines and vitamin $\mathrm{D}$.

\section{References}

1 World Health Organization. Global report on diabetes. Suiza; 2016. p. 1-84.

2 Buhary BM, Almohareb O, Aljohani N, Alrajhi S, Elkaissi S, Sherbeeni S, et al. Association of Glycosylated Hemoglobin Levels With Vitamin D Status. J Clin Med Res. 2017;9(12):1013-8.

3 Williams A, Greene N, Kimbro K. Cytokine Increased circulating cytokine levels in African American women with obesity and elevated HbA1c. Cytokine. 2020; 128:154989.

4 Joergensen C, Gall M-A, Schmedes A, Tarnow L, Parving H-H, Rossing P. Vitamin D Levels and Mortality in Type 2. Diabetes Care. 2010;33(10):2238-42.

\section{$451 / 41$}

\section{Interplay between Dendritic and T Cells in Response} to Membrane Vesicles from Gut Beneficial Microbes

\author{
Diaz, N. ${ }^{1}$; Cordero Alday, C. ; Olivo, Y. ${ }^{1}$; Badía Palacín, J. ${ }^{2}$; \\ Baldomà Llavinés, L. ${ }^{3}$ \\ ${ }^{1} \mathrm{PhD}$ student; ${ }^{2} \mathrm{PP} 1 ;{ }^{3} \mathrm{PP} 2$
}

Interconnection between nutrients and gut microbes is a key issue governing the healthy/pathological status of an individual [1]. Probiotics are a nutritional strategy for restoring a healthy microbiota community. However, there is still much to learn about the mechanisms of probiotic action [2,3]. All bacteria release membrane vesicles (MVs) as a mechanism of intercellular communication [4]. This study aims at evaluating the role of gut microbiota MVs in the modulation of host immune responses and characterize the effector molecules involved. We used different cellular models of immune cells. Monocytes from healthy donors were differentiated into DCs for 7 days and then stimulated with MVs from the probiotic Nissle $1917(\mathrm{EcN})$ and the commensal ECOR12. Stimulated DCs were co-cultured with T cells in two different models: (i) direct contact with CD4+ T cells, (ii) indirect contact with CD4+ naïve T cells. Immune responses were analyzed by flow cytometry and cytokine quantification. DC-derived exosomes were isolated and structurally characterized. Their surface markers were inspected with MACSplex Exosome kit. Results showed MVs from both strains induced maturation of DCs with different IL-10, IL-6, and TNF- $\alpha$ secretion profiles. After 6-day coculture (mDCs/Tcells), activated DCs induced polarization of LTs towards Th1/Th17/Th2 responses in a strain-specific manner. Exosomes isolated from DCs displayed similar size but different surface marker content. This study confirms that microbiota MVs modulate DC function. Activated DCs can drive Treg/Th specific responses by mechanisms that depend not only on direct contact through DCs but also on soluble factors including cytokines and exosomes.

Acknowledgments: ND acknowledges her doctoral fellowship from Agencia Nacional de Investigación y Desarrollo (ANID)/ Scholarship Program/DOCTORADO BECAS CHILE /201772180379. CCA acknowledges her doctoral fellowship from Agencia Nacional de Investigación y Desarrollo (ANID)/ Scholarship Program/DOCTORADO BECAS CHILE/202072210404. YO acknowledges her doctoral fellowship from Ministerio de Ciencia, Tecnología e innovación (Minciencias) COLOMBIA/Scholarship INTERNATIONAL/2020-860.

Program/DOCTORADO

Financial Support: This research was funded by Ministerio de Ciencia e Innovación, Gobierno de España, grant number PID2019-107327RB-100.

Conflict of Interest: None.

Authorship: Conceptualization, JBP and LBL; investigation, ND, CCA, YO, LBL and JBP; analysing the data: ND; writingoriginal draft preparation, ND; writing - review and editing, LBL and JBP; supervision, LBL; project administration, JBP; funding acquisition, LBL and JBP.

Keywords: Immune interplay, probiotics, dendritic cells, exosomes, bacterial membrane vesicles.

\section{References}

1. Belkaid, Y.; Hand, T.W. Role of the microbiota in immunity and inflammation. Cell 2014, 157, 121-141, doi: 10.1016/j.cell.2014.03.011.

2. Shanahan, F. The gut microbiota in 2011: Translating the microbiota to medicine. Nat Rev Gastroenterol Hepatol, 2011, 9(2), 72-74. doi:10.1038/ nrgastro.2011.250

3. Suez, J.; Zmora, N.; Segal, E.; Elinav, E. The pros, cons, and many unknowns of probiotics. Nat. Med. 2019, 25, 716-729, doi:10.1038/s41591019-0439-x.

4. Kaparakis-Liaskos, M.; Ferrero, R.L. Immune modulation by bacterial outer membrane vesicles. Nat Rev Immunol 2015, 15, 375-387, doi:10.1038/nri3837. 
$451 / 42$

\section{Systemic Pro-Inflammatory Cytokines' Level Following 7-Day High-Salt Diet in Healthy Young Individuals}

Mihaljević, Z. ${ }^{1}$; Knezović, A. ${ }^{2}$; Šušnjara, $P .{ }^{1}$; Mihalj, M. ${ }^{1}$; Drenjančević, I. ${ }^{1}$; Stupin, $A .{ }^{1}$

${ }^{1}$ Department of Physiology and Immunology, Faculty of Medicine J.J. Strossmayer University of Osijek; ${ }^{2}$ Community

Health Center Osijek-Baranja County, HR-31000 Osijek, Croatia

Present study aimed to test the effect of 7-day high-salt (HS) diet on pro-inflammatory cytokines' serum concentration, and to examine the potential association between the level of systemic inflammation and previously reported impaired microvascular endothelial function due to HS loading in young healthy individuals (1-5). Twenty four young healthy individuals (12 men and 12 women, age ranged 18-26) were assigned to 7-day low-salt (LS) diet ( $<3.5 \mathrm{~g}$ salt/day) ("wash-out" period) followed by 7 -day HS diet ( $\sim 14 \mathrm{~g}$ salt/day). Serum protein concentration of interferon gamma (IFN $\gamma)$, interleukin 6 (IL-6) and tumor necrosis factor alpha (TNF- $\alpha$ ) was measured by commercially available panels for multiplex protein quantitation (Luminex 200 instrument platform) before and after HS. Skin microvascular blood flow in response to vascular occlusion (post-occlusive reactive hyperemia; $\mathrm{PORH}$ ) and iontophoresis of acetylcholine (ACh-induced dilation; AChID) was assessed by laser Doppler flowmetry at LS and HS. Changes in calculated daily salt intake from $24 \mathrm{~h}$ sodium excretion confirmed that participants conformed to the diet protocols. Serum concentration of IFN $\gamma$, IL- 6 and TNF- $\alpha$ significantly increased, while PORH and AChID were significantly impaired following HS diet compared to LS. Salt intake significantly positively correlated with increased IFN $\gamma$, while there was no correlation between changes in pro-inflammatory cytokine serum concentrations and microvascular reactivity due to 7-day HS loading. Present results suggest that 7-day HS diet can promote systemic inflammation in young healthy individuals. However, it remains to establish whether more specific changes in inflammatory response directly contribute to the impaired microvascular endothelial function following HS diet.

Acknowledgments: None.

Financial Support: The present study was supported by Croatian Science Foundation under the project \#IP-2016-06-8744 Interaction of Renin-Angiotensin and Adrenergic System in Oxidative Stress Induced Endothelial Activation (RAS-AdrenOX).

Conflict of Interest: None.

Authorship: Formulating the research question: MM, ID and AS. Designing the study: MM, ID and AS. Carrying study out: ZM, AK, PS and AS. Analyzing the data: ZM and AS. Writing the article: ZM, MM, ID and AS.

Keywords: High-salt diet, inflammation, endothelium, microcirculation, cytokines.

\section{References}

1 Barić, L, Drenjančević, I, Mihalj, M et al. (2020) Enhanced Antioxidative Defense by Vitamins C and E Consumption Prevents 7-Day High-Salt Diet-Induced Microvascular Endothelial Function Impairment in Young Healthy Individuals. J Clin Med 9(3), 843.

2 Mihalj, M, Matić, A, Mihaljević, $Z$ et al. (2019) Short-Term High-NaCl Dietary Intake Changes Leukocyte Expression of VLA-4, LFA-1, and Mac-1 Integrins in Both Healthy Humans and Sprague-Dawley Rats: A Comparative Study. Mediators Inflamm 2019, 6715275.

3 Barić, L, Drenjančević, I, Matić, A et al. (2019) Seven-Day Salt Loading Impairs Microvascular Endothelium-Dependent Vasodilation without Changes in Blood Pressure, Body Composition and Fluid Status in Healthy Young Humans. Kidney Blood Press Res 44(4), 835-847.

4 Cavka, A, Jukic, I, Ali, M et al. (2016) Short-term high salt intake reduces brachial artery and microvascular function in the absence of changes in blood pressure. J Hypertens 34(4), 676-84.

5 Cavka, A, Cosic, A, Jukic, I et al. (2015) The role of cyclo-oxygenase-1 in high-salt diet-induced microvascular dysfunction in humans. J Physiol 593(24), 5313-24.

\section{$451 / 43$}

\section{An Antioxidant-Enriched Diet is Beneficial in Alzheimer Disease Triple Transgenic Mice but Harmful in Non-Transgenics}

\author{
Cepria, N. ${ }^{1}$; Garcia-Solis, B. ${ }^{1}$; Diaz-Del Cerro, E. ${ }^{1}$; Gimenez-Llort, L. ${ }^{2}$; \\ de la Fuente, M. $^{1}$ \\ 'Department of Genetics, Physiology and Microbiology, \\ Faculty of Biology, Complutense University of Madrid, Spain; \\ ${ }^{2}$ Department of Psychiatry and Forensic Medicine, Institute of \\ Neurosciences, Autonomous University of Barcelona, Spain
}

The immune system and the oxidative stress play a key role in aging and aged-related diseases, such as Alzheimer's Disease $(1,2)$. Thus, the ingestion of antioxidant-enriched diets has been used as a strategy to improve the aging process and these diseases, in old individuals (3). Nevertheless, the positive effects of this intervention in young animals are unknown. Therefore, our aim was to study the effects, in several immune functions, oxidative stress parameters and in longevity, of an antioxidant-enriched diet ingested by triple-transgenic mice for Alzheimer Disease (3xTgAD) and non-transgenic mice (NTg) from young age. For this, NTg and $3 \times$ TgAD female mice, received, from 2.5 months of age, an enriched diet with $0.1 \%$ of thiaproline and $\mathrm{N}$-acetylcysteine, (NTgD and $3 x$ TgADD, respectively) or a standard diet (NTgC, 3xTgADC). At 4, 6 and 12-months, peritoneal leukocytes were collected and function (phagocytosis, natural killer activity, lymphoproliferative response) and oxidative stress (oxidized glutathione, reduced glutathione, xanthine oxidase activity) parameters were measured. Diet supplementation was maintained until the natural death of mice, and the lifespan were recorded. The results showed and important alteration in the functions of peritoneal leukocytes of the NTgD compared to NTgC. Meanwhile, the 3xTgADD mice showed an improvement in some functions compared to 3xTgADC but did not reach those values of NTgC mice. The NTgD mice showed the lowest lifespan, while the $3 \times$ TgADD mice showed a similar lifespan to NtgC. In conclusion, this antioxidant diet, 
ingested very early in life, may be beneficial in pathological subjects, but can be harmful in non-pathological conditions.

Acknowledgments: We would like to thank Ianire Maté, Julia Cruces and Antonio Garrido for their technical assistance.

Financial Support: FIS-(PI15/01787)-ISCIII-FEDER.

Conflict of Interest: None.

Authorship: Conceptualization, NC, LG-L and MdF; Formal analysis, NC; Methodology and investigation, NC, BG-S, ED-DC. $\mathrm{NC}$ and $\mathrm{MdF}$ redacting the draft.

Keywords: Antioxidants; 3xTgAD mice; immune functions; oxidative stress.

\section{References}

1 Gella, A., Durany, N. (2009) Oxidative stress in Alzheimer disease. Cell Adh Migr 3, 88-93. doi:10.4161/cam.3.1.7402.

2 Heppner, F.L., Ransohoff, R.M., Becher, B. (2015) Immune attack: the role of inflammation in Alzheimer disease. Nat Rev Neurosci 16, 358-372. doi:10.1038/nrn3880.

3 Luchsinger, J.A., Mayeux, R. (2004) Dietary factors and Alzheimer's disease. Lancet Neurol 3, 579-587. doi:10.1016/s1474-4422(04)00878-6.

\section{$451 / 44$ \\ Akkermansia muciniphila Ameliorates the Age-Related Impairment of Immune Function in Old Mice}

Ceprian, N. '; Diaz-Del Cerro, E. ${ }^{2}$; Lambea, M. ${ }^{2}$; Salazar Garzo, N. ${ }^{3}$; Gueimonde, M. ${ }^{3}$; de la Fuente, M. ${ }^{2}$

${ }^{1}$ Dept. Genetics, Physiology and Microbiology. Biology Faculty. Complutense University of Madrid; ${ }^{2}$ Dept. Genetics, Physiology, Microbiology (Animal Physiology Unit). Complutense University of Madrid; ${ }^{3}$ Dept. of Microbiology and Biochemistry of Dairy Products, IPLA-CSIC

Immune system, a homeostatic system, deteriorates during aging as consequence of the establishment of an oxidative stress, leading to higher frequency of infections and tumors with the advancement of age (1). Currently, the number and proportion of the elderly people in the world is increasing as well as the disabilities and diseases associated with age and, consequently, the search for lifestyle strategies in order to slow down aginghas increased $(1,2)$. In this context, knowing the important role of the gut microbiota in health, and consequently in the rate of aging, the use of probiotics is being considered in recent years (2). The "new" probiotic Akkermansia muciniphila can be very promising since its intestinal concentration decreases with advancing age but increases in centenarians (3). However, its benefit effect on immune functions in old individuals is unknown. Thus, our aim was to investigate the effect of daily intake of $A$. muciniphila (108 CFU/100 $\mu \mathrm{L}$ PBS), for one month, on several parameters of immune function in peritoneal immune cells of ICR-CD1 old ( $74 \pm 4$ weeks of age) female mice. The results showed that old animals that ingested $A$. muciniphila, compared to those of their age who did not take it, significantly improved their chemotaxis of lymphocytes and macrophages $(\mathrm{p}<0.05)$, Natural Killer activity $(\mathrm{p}<0.001)$ and, CoAand LPS-stimulated lymphoproliferation $(\mathrm{p}<0.01$ and $\mathrm{p}<0.05)$. In conclusion, since the immune functions studied are markers of health and of the rate of aging, the daily supplementation with $A$. muciniphila could be proposed as a useful strategy to improve aging and achieve healthy longevity.

Acknowledgments: We would like to thank Judith Felix and Irene Martinez de Toda for their technical assistance.

Financial Support: This research did not receive any specific grant from funding agencies in the public, commercial, or not-forprofit sectors.

Conflict of Interest: None.

Authorship: MdF and MG were responsible for the design of the study. ED-DC carried out all the experiments. ML and NC helped in performing several of the assays. ED-DC took part in the statistical analysis. NSG helped producing and administrating the probiotic studied.

Keywords: Aging, Immune functions, probiotics, Akkermansia muciniphila, mice.

\section{References}

1 De la Fuente M, Miquel J. (2009) An update of the oxidation-inflammation theory of aging: the involvement of the immune system in oxi-inflammaging. Curr Pharm Des 15(26), 3003-26. doi: $10.2174 / 138161209789058110$

2 De la Fuente M., Diaz-Del Cerro E., Salazar N. et al. (2020) "Microbiota and probiotics in aging". Approaches to Aging Control. 24, 127-142.

3 Derrien M, Vaughan EE, Plugge CM. et al. (2004) Akkermansia municiphila gen. nov., sp. nov., a human intestinal mucin-degrading bacterium. Int J Syst Evol Microbiol 54, 1469-1476. doi: 10.1099/ijs.0.02873-0.

\section{$451 / 45$}

\section{Remission of Systemic Lupus Erythematosus, Scleroderma and Pulmonary Fibrosis with Micronutritional Medicine}

\author{
Cardona Burrull, F. ${ }^{1}$; Blanco Rogel, M. ${ }^{2}$; Capdevila Menac, N. ${ }^{3}$ \\ ${ }^{1}$ Cardiology and Pneumology Department. SAR; ${ }^{2}$ Barcelona \\ Oficial Pharmacy College; ${ }^{3}$ University of Barcelona
}

Systemic lupus erythematosus and scleroderma are chronic systemic autoinmune diseases with a broad spectrum of skin and internal organs affectations $(1,2,3)$. They can occur simultaneously in the same patient (overlap syndrome) $(4,5)$ and frequently they begin to manifest with the Raynaud phenomenon.

We present a 55-year-old woman with overlap syndrome and pulmonary fibrosis with pulmonary hypertension. She presents multiple symptoms with a great impact in her quality of life. Chronic autoimmune diseases share a common pattern of excess oxidation and inflammation that results from mitochondrial alteration (6-8) and a parallel decrease in glutathione (9) with a wide spectrum of micronutrient deficiencies $(10,11)$. Progressive drug deprescription was achieved, leaving only escitalopram of the eight initial drugs. A micronutritional aboradage was started to reduce excess of chronic inflammation and oxidative stress with a blend 
of vitamins (coenzyme), minerals (cofactors), coenzyme Q10 and R-lipoic acid with specific fatty acids omega 3 ( $\alpha$ - linolenic acid, docosahexaenoic acid) (10) and omega 6 ( $\Upsilon$ linolenic acid) (12). Other specificmicronutrients were supplemented: $\mathrm{N}$-acetylcysteine to increase intracellular glutathione and reduce fibroblast proliferation, replenish celular thiols and inhibit collagen synthesis $(13,14)$; N-acetylglucosamine $(15,16)$ and quercetin $(17,18)$ to decrease immune hyperresponsiveness and improve intestinal integrity together with nucleotides; Free amino acids, choline (19) and myo-inositol (20) to support mitochondrial function and metabolic disturbances (19).

Micronutritional medicine and appropriate dietary guidellines (low carb, antiinflamatory and animal protein restriction) may alleviate the multiple symptoms associated with autoimmune disease with progressive drug deprescription. This intervention allows her to regain the ability to live independently together with a great improvement in her quality of life.

Acknowledgments: Special study personnel, patients and their families.

Financial Support: None.

Conflict of Interest: FCB declares no conflict of interest. MBR has received consulting and advisor fees from Laboratorio de Complementos Nutricionales S.L. NCM is a LCN lab employee in the medical writing area.

Authorship: FCB developed procedures for paper selection and data extraction.

MBR and NCM contributed to the analysis and interpretation of data and review of this case report. All authors have approved the final draft for submission.

Keywords: Systemic lupus erythematosus, scleroderma, pulmonary fibrosis, inmunonutrients, micronutrients.

\section{References}

1 Di Battista M, Marcucci E, Elefante E, Tripoli A, Governato G, Zucchi D, et al. One year in review 2018: Systemic lupus erythematosus. Clin Exp Rheumatol. 2018 Sep;36(5):763-77.

2 Ezquerra Gadea J, López Larrayoz I, García Cores F, García Muñoz M. Fenómeno de Raynaud y esclerodermia. Atención Primaria. 2003;32(1):60.

3 Sociedad Española de Reumatología (SER). Manual SER de diagnóstico y tratamiento de las enfermedades reumáticas autoinmunes sistémicas. Primera. Vol. 1, Elsevier. Barcelona; 2014.

4 Ezquerra Gadea J, López Larrayoz I, García Cores F, García Muñoz M. Fenómenode Raynaudy esclerodermia. Atención Primaria.2003;32(1):60

5 Aspe Unanue L, González Hermosa MR, Gardeazabal García J. Scleroderma (systemic sclerosis). Piel. 2010;25(5):252-66.

6 Agrawal A, Mabalirajan U. Rejuvenating cellular respiration for optimizing respiratory function: Targeting mitochondria [Internet]. Vol. 310, American Journal of Physiology - Lung Cellular and Molecular Physiology. American Physiological Society; 2016. p. L103-13.

7 Birch J, Barnes PJ, Passos JF. Mitochondria, telomeres and cell senescence: Implications for lung ageing and disease. Vol. 183, Pharmacology and Therapeutics. Elsevier Inc.; 2018. p. 34-49.

8 Murphy MP, Hartley RC. Mitochondria as a therapeutic target for common pathologies. Vol. 17, Nature Reviews Drug Discovery. Nature Publishing Group; 2018. p. 865-86.

9 Yang J, Yang X, Zou H, Li M. Oxidative Stress and Treg and Th17 Dysfunction in Systemic Lupus Erythematosus. Vol. 2016, Oxidative Medicine and Cellular Longevity. Hindawi Limited; 2016.
10 Islam MA, Khandker SS, Kotyla PJ, Hassan R. Immunomodulatory Effects of Diet and Nutrients in Systemic Lupus Erythematosus (SLE): A Systematic Review. Front Immunol. 2020;11:1477.

11 Constantin MM, Nita IE, Olteanu R, Constantin T, Bucur S, Matei C, et al. Significance and impact of dietary factors on systemic lupus erythematosus pathogenesis (Review). Exp Ther Med. 2019;17(2):1085-90.

12 Olveira G, Olveira C, Acosta E, Espíldora F, Garrido-Sánchez L, GarcíaEscobar E, et al. La suplementación con ácidos grasos mejora parámetros respiratorios, inflamatorios y nutricionales en adultos con fibrosis quística. Arch Bronconeumol. 2010;46(2):70-7

13 Grygiel-Górniak B, Puszczewicz M. Oxidative Damage and Antioxidative Therapy in Systemic Sclerosis. Mediators Inflamm. 2014;2014:1-11.

14 Doridot L, Jeljeli M, Chêne C, Batteux F. Implication of oxidative stress in the pathogenesis of systemic sclerosis via inflammation, autoimmunity and fibrosis. Redox Biol [Internet]. 2019;25:101122.

\section{$451 / 46$ \\ Vitamin D Concentration and its Impact on Immunity in Polish indoor and outdoor athletes}

\author{
Orysiak, J. '; Malczewska-Lenczowska, J.2.; Młynarczyk, M. '; \\ Szczepańska, B. ${ }^{2}$; Surała, O. ${ }^{2}$ \\ ${ }^{1}$ Department of Ergonomics, Central Institute for Labour \\ Protection - National Research Institute; ${ }^{2}$ Department of \\ Nutrition Physiology and Dietetics, Institute of Sport - National \\ Research Institute
}

The majority of people do not have the optimal level of vitamin D $(1,2)$. Vitamin D may regulate both adaptive and innate immunity $(3,4)$. The study aimed to examine vitamin $\mathrm{D}$ concentration in ice and field hockey players before and after the summer sun exposure (without supplementation) and its impact on immunity. Male ice hockey $(\mathrm{IH} ; \mathrm{n}=19)(5)$ and field hockey $(\mathrm{FH} ; \mathrm{n}=12)$ players participated in the study. Blood and saliva samples were collected twice (before and after the summer sun exposure) to examine total 25-hydroxyvitamin D (25(OH)D) concentration, white blood cell and its population counts in blood, and secretory immunoglobulin A ( $\operatorname{sgA})$ concentration in saliva. $25(\mathrm{OH}) \mathrm{D}$ concentration significantly increased after summer in $\mathrm{FH}$ and $\mathrm{IH}$ players $(\mathrm{FH}: 26.7 \pm 4.4$ $\mathrm{ng} / \mathrm{ml}$ and $33.6 \pm 4.8 \mathrm{ng} / \mathrm{ml} ; \mathrm{IH}: 26.5 \pm 3.1 \mathrm{ng} / \mathrm{ml}$ and $31.3 \pm 3.1 \mathrm{ng} / \mathrm{ml}$ before and after sun exposure, respectively). Vitamin D concentration did not differ between outdoor and indoor athletes. There were no statistically significant correlations between immune markers and 25( $\mathrm{OH}) \mathrm{D}$ concentrations in $\mathrm{IH}$ and $\mathrm{FH}$ players in two occasion. Immune markers also did not differ between deficient $(<30 \mathrm{ng} / \mathrm{ml})$ and sufficient $(>30 \mathrm{ng} / \mathrm{ml})$ vitamin $\mathrm{D}$ status groups. In conclusion, after the summer sun exposure, vitamin D levels increased in all athletes. However, the obtained concentrations appear to be insufficient to maintain optimal vitamin $\mathrm{D}$ levels during the autumn-winter period. $25(\mathrm{OH}) \mathrm{D}$ concentration was not associated with immune markers. Although vitamin D status was not linked to immunity in this study, other negative effects of its deficiency on health can not be ruled out (4).

Acknowledgments: The authors thank the athletes and coaching staff who contributed to this project.

Financial Support: This study received financial support from the Ministry of Sport and Tourism (grant number: 2016.037/40/ $\mathrm{BP} / \mathrm{DSW}$ ) in the Republic of Poland.
18

Ann Nutr Metab

DOI: $10.1159 / 000518127$ 
Conflict of Interest: None.

Authorship: JO, JM-L - formulating the research question(s); JO, JM-L - designing the study; JO, JM-L, BS - carrying it out; JO, MM, JM-L, OS - analysing the data; JO, MM, JM-L, OS, BS writing the article.

Keywords: Athletes, vitamin D, sun exposure, secretory immunoglobulin A, saliva.

\section{References}

1 Aydın CG, Dinçel YM, Arıkan Y, et al. (2019) The effects of indoor and outdoor sports participation and seasonal changes on vitamin D levels in athletes. SAGE Open Med 7, 2050312119837480.

2 Rusińska A, Płudowski P, Walczak M, et al. (2018) Vitamin D Supplementation Guidelines for General Population and Groups at Risk of Vitamin D Deficiency in Poland-Recommendations of the Polish Society of Pediatric Endocrinology and Diabetes and the Expert Panel With Participation of National Specialist Consultants and Representatives of Scientific Societies-2018 Update. Front Endocrinol (Lausanne) 9, 246.

3 Greiller CL \& Martineau AR. (2015) Modulation of the immune response to respiratory viruses by vitamin D. Nutrients7, 4240-4270.

4 Orysiak J, Fitzgerald JS, Malczewska-Lenczowska J, et al. (2020) Vitamin $\mathrm{D}$ and upper respiratory tract infections in young active males exposed to cold environments. Ann Agric Environ Med doi:10.26444/ aaem $/ 127530$.

5 Crewther B, Cook C, Fitzgerald J, et al. (2020) Vitamin D and Cortisol as Moderators of the Relationship Between Testosterone and Exercise Performance in Adolescent Male Athletes. Pediatr Exerc Sci doi: 10.1123/ pes.2019-0229. Online ahead of print.

\section{$451 / 48$}

\section{Immunonutritional Supplements for Precision Nutrition at Controlling Infectious and Inflammatory Processes}

Laparra Llopis, J. '; Bouzas Muñoz, A. ${ }^{2}$; Gomez De Cedron, M. ${ }^{3}$; Reglero, $\mathrm{G}^{3}{ }^{3}$, Ramírez, A. $^{3}$

${ }^{1}$ Madrid Institute for Advanced Studies in Food (Imdea Food); ${ }^{2}$ Imdea Food / Canaan Research \& Investment Group,

${ }^{3}$ Imdea Food

This study aimed to evaluate the role of immunonutritional Rosmarinus officinalis (RE)-based supplements at promoting a selective functional differentiation of human macrophage-like cells. To this end, cell cultures were challenged to increasing dose concentrations (1/2IC50 and IC50) and the FACS immunephenotyping was assessed. It was observed a dose-dependent effect on macrophages' M1/M2 polarization phenotypes. It is noteworthy that phenotypic control in the macrophage population is not associated with the expression of CD206, indicating the non-development of an immunosuppressive population that could favor the progression of the COVID-19 infection. The immunophenotypic changes observed in the macrophage population are accompanied by adaptations in their metabolic capacity (oxygen consumption rates measured by Seahorse $\odot$ technology). Exposure to RE favors a greater reserve respiratory capacity (Spare Resp), derived from a greater use of fatty acids (non-mito OCR) by the macrophage population. Importantly, this is associated with an increase in the proportion of mediators controlling the inflammatory response, such as lysophosphatidylcholine and the inflammasome inhibitor (NRLP3), which translates into a more controlled inflammatory response as indicated by the lower expression of CD68. The involvement of the activation of the TLR4 receptor, or associated pathways, is evidenced by the change of direction in the response after exposure to C34 (TLR4 inhibitor) and its prototypical agonist (i.e., lipopolysaccharide-LPS). Thus, it is concluded that RE-based immunonutritional supplements can help to improve the quality of innate immune response(s) during infection associated processes.

Acknowledgments: JLL thanks MICINN for his 'Ramon y Cajal' contract (RYC-2015-18083). ABM thanks the industrial predoctoral program of Community of Madrid for funding his research IND2017/BIO-7857).

Financial Support: This work was financially supported by grant RYC-2015-18083 and Food4ImNut [PID2019107650RB-C22, Ministry of Science, Innovation and Universities MICIU]. This work was supported by a grant from the industrial predoctoral program of the Community of Madrid (IND2017/ BIO-7826), Plan Nacional I + D + i PID2019-110183RB-C21; the Regional Government of Community of Madrid P2018/BAA4343-ALIBIRD2020-CM; Ramon Areces Foundation; EU Structural Funds, and COST Action CA17118.

Conflict of Interest: The authors declare that they have no conflict of interest.

Authorship: All authors have contributed equally to formal analysis, data curation and writing and revising the document, AR and JLL also contributed to managing funding resources.

Keywords: Rosmarinus officinalis, innate immunity, macrophages, TLR4, nutritional supplements.

\section{References}

N/A
$451 / 49$

Effect of Mediterranean Diet on the Disability of Hospitalized Older Adults Following an Exercise Intervention

\author{
Tor Roca, A. ' ; Mayordomo-Cava, J. ${ }^{2}$; Andrés-Lacueva, C. ${ }^{1}$; \\ Serra-Rexach, J. ${ }^{2}$; Urpí-Sardà, M. ${ }^{1}$ \\ ${ }^{1}$ Biomarkers and Nutrimetabolomics Laboratory. University \\ of Barcelona, Barcelona, Spain.; ${ }^{2}$ Research Health Institute, \\ Gregorio Marañon Hospital, Madrid, Spain
}

In this study we aimed to investigate the relation between adherence to a Mediterranean dietary (MedDiet) pattern and disability in acutely hospitalized very old patients in the context of a simple inpatient exercise program (NTC 03604640). The mean (SD) age of participants $(n=108)$ was $87.74(4.57)$ and $45 \%$ were women. The adherence to a MedDiet pattern was measured with MEDAS questionnaire (1). In the hospital admission, patients in 
the highest tertile of MedDiet adherence pattern showed higher Barthel score $(p$-values $=0.03$ ) compared to those in the other lower tertiles. After admission, participants were randomized into two groups: a control group ( $\mathrm{n}=45$, usual care) and an intervention group ( $n=63$, supervised exercise, i.e., walking and rising from a chair [1-3 sessions/day]). The meantime (SD) of hospitalization was 6.60 (7.02) days. Preliminary results of this ongoing study at discharge time $(n=94)$ showed that patients in the control group and lowest tertile of MedDiet adherence seems to have lower Barthel score (mean (SD): 56.7 (28.7)) compared to those in the highest tertile (mean (SD): $72.3(24.2))(\mathrm{p}=0.12)$ and to those in the intervention group and same tertile (mean (SD): 75.8 (19.5) $(p=0.07)$ ). In conclusion, the higher adherence to MedDiet could possibly have a similar effect on Barthel score than an intervention with exercise in these patients. These preliminary results showed the positive role of MedDiet, as immunomodulatory and antiinflammatory among other properties, on functional status.

Acknowledgments: We thank the geriatrics ward team from Hospital General Universitario Gregorio Marañón (Madrid, Spain) for their valuable help and contribution to our results.

Financial Support: This research was supported by the EU Joint Programming Initiative 'A Healthy Diet for a Healthy Life'; ERA-Net cofund on Intestinal Microbiomics (INTIMIC) Project "AC19/00096", PI17/02021 and PI18/00139 by Instituto de Salud Carlos III and co-funded by European Regional Development Fund "A way to make Europe"; CIBERFES (co-funded by the FEDER Program from EU); 2017 SGR1546 grant from the Generalitat de Catalunya Agency AGAUR. ATR is grateful for the FPU 2019 (Ministerio de Educación y Formación Profesional) contract and CAL to the ICREA Academia award 2018 from the Generalitat de Catalunya.

Conflict of Interest: None.

Authorship: J.M.-C., JS-R, C.A.-L., and M.U.-S. formulated the research question and designed research; J.M.-C., and JS-R carried out research; ATR, J.M.-C., and M.U.-S. analysed data and performed statistical analysis; ATR, and M.U.-S. wrote the paper; J.M.-C., JS-R, and C.A.-L. critically reviewed this abstract. All the authors read and approved the final abstract.

Keywords: Mediterranean diet, disability, elderly, activities of daily living, physical activity.

\section{Reference}

1 Schröder $\mathrm{H}$ et al. A short screener is valid for assessing Mediterranean diet adherence among older Spanish men and women. J Nutr. 2011;141(6):1140-5. doi: 10.3945/jn.110.135566.

\section{$451 / 50$ \\ Shotgun Proteomics for the Characterization of Quinoa Seeds: A Promising Tool for Nutritional Studies}

Galindo Luján, R. ${ }^{1}$; Pont, L. ' ; Minic, Z. ${ }^{2}$; Berezovski, M. ${ }^{2}$; Sanz-Nebot, V. ${ }^{1}$; Benavente, $F{ }^{1}$

${ }^{1}$ University of Barcelona; ${ }^{2}$ University of Ottawa

Quinoa (Chenopodium quinoa Willd.) is an Andean grain that has been progressively introduced in western countries as a protein-rich super food. Because of its attractive nutritional composition, mainly based on the presence of lipids, carbohydrates, polyphenols, fiber and especially proteins, it is being investigated as a promising source for the development of functional foods and nutraceutical products [1]. Proteins derived from quinoa seeds are supposed to present bioactivities towards the promotion of wellbeing and disease prevention in humans [2]; therefore, there is an urgent need to develop novel analytical methods to get further information about the quinoa proteome, which could be the key tool to improve the understanding of the mechanisms involved in many human biological responses. In this study, a label-free LC-MS/MS shotgun proteomics approach with a state-of-the-art Orbitrap mass spectrometer was applied to comprehensively characterize the proteome of the most typical quinoa seed varieties, i.e. black, red, white quinoa from Peru and white quinoa from Bolivia (also referred to as royal). After carefully selecting the most appropriate conditions (regarding sample preparation, instrumentation and data analysis) a total of 1,211 quinoa proteins were identified. Inspection and visualization using Venn diagrams, heat maps and Gene Ontology (GO) graphs revealed proteome similarities and differences between the four varieties. The presented data provides the most comprehensive experimental quinoa seed proteome map existing to date in the literature, as a starting point to perform more specific characterization and nutritional studies of quinoa and quinoa-containing foodstuff.

Acknowledgments: Cathedra UB Rector Francisco Buscarons Úbeda (Forensic Chemistry and Chemical Engineering).

Financial Support: This study was supported by the Spanish Ministry of Economy and Competitiveness (RTI2018-097411-B-I00).

RGL thanks the National Agricultural Innovation Program and the Ministry of Education from Peru for a research stay and a $\mathrm{PhD}$ fellowship, respective.

Conflict of Interest: The authors declare no conflict of interest. Authorship: FB.

Keywords: LC-MS/MS; label-free quantification; quinoa; shotgun proteomics.

\section{References}

1 Vilcacundo, R., \& Hernández-Ledesma, B. (2017). Nutritional and biological value of quinoa (Chenopodium quinoa Willd.). Current Opinion in Food Science, 14, 1-6.

2 Capraro, J., De Benedetti, S., Di Dio, M., Bona, E., Abate, A., Corsetto, P. A., \& Scarafoni, A. (2020). Characterization of chenopodin isoforms from quinoa seeds and assessment of their potential anti-inflammatory activity in caco-2 cells. Biomolecules, 10, 1-15. 


\section{$451 / 51$}

Vitamin-D Deficiency is More Common in UK Black People and a Possible COVID-19 Risk Factor

Sobczynska-Malefora, A. ; Harbige, L. ${ }^{2}$; Steed, D. ${ }^{3}$; MacMahon, E. ${ }^{4}$; Shankar-Hari, M. ${ }^{4}$; Harrington, D. ${ }^{1}$

'The Nutristasis Unit, Viapath, St. Thomas' Hospital, London; King's College London; ${ }^{2}$ Lipidomics and Nutrition Research Centre, London Metropolitan University, London, UK;

${ }^{3}$ Application Management Services, Viapath, St. Thomas' Hospital, London, UK; ${ }^{4}$ GSTT NHS Foundation Trust, London, UK; King's College London, London, UK

Introduction: Risk and severity of SARS-CoV-2 infection has been linked to vitamin D inadequacy (1). Vitamin D inadequacy is prevalent in older adults, Black and Asian population groups, and those with high BMI (2). These populations disproportionately account for the majority of critically ill UK COVID-19 patients (3).

Objectives: To test the association between serum 25hydroxyvitamin D (25(OH)D) with ethnicity, sex, BMI and laboratory data in all samples received between January-June 2020, St. Thomas' Hospital, N=17628 and SARS-CoV-2 RNA/IgG screening (March 2020-January 2021).

Results: $25(\mathrm{OH}) \mathrm{D}$ deficiency $(<25 \mathrm{nmol} / \mathrm{L})$ was $25 \%, 21 \%$ and $17 \%$; and insufficiency $(25-50 \mathrm{nmol} / \mathrm{L}) 36 \%, 34 \%$ and $33 \%$ in Blacks, Asians and Whites respectively. A $18.5 \%$ of females had $25(\mathrm{OH}) \mathrm{D}<25 \mathrm{nmol} / \mathrm{L}$ compared with $22.4 \%$ males. There was no sex difference in BMI and age in Whites, but significant BMI and age differences between the sexes in Blacks. The levels of $25(\mathrm{OH}) \mathrm{D}$ differed between sex in Blacks, Whites and Chinese Asians but not in Asian Indians. Four hundred and eighty five (2.7\%) (median/ IQR 25(OH)D $42 \mathrm{nmol} / \mathrm{L}$ (25-66)) patients had COVID-19 within this cohort. One hundred and seventeen $(24.1 \%)$ of these had The levels of 25(OH)D deficiency and $178(36.7 \%)$ insufficiency (total $60.8 \%$ deficient/insufficient). Importantly $42 \%$ of those with deficiency were black people. In a sub-set cohort $(n=45)$ of COVID-19 patients, 25 $(\mathrm{OH}) \mathrm{D}$ correlated negatively with inflammation as measured by TNF- $\alpha$ (Pearson's $r=-0.395, p<0.01$ ) consistent with $25(\mathrm{OH}) \mathrm{D}$ 's role in modulating levels of pro-inflammatory cytokines (4).

Conclusions: Highest prevalence of COVID-19 was among black people previously found 25(OH)D deficient. 25(OH)D deficiency maybe an important and preventable risk factor for COVID19 and associated hyperinflammation.

Acknowledgments: The authors are thankful to staff from the Clinical Research and Development (King's College Hospital) and Laboratory Informatics Team (Viapath and GSTT) for their support of this work.

Financial Support: None.

Conflict of Interest: The authors declare no conflict of interest.

Authorship: LH: study concept, AS-M, DH and DS: design, DS and AS-M: acquisition, AS-M: database management and statistical analysis, AS-M, LH, EMM, MS-H: interpretation, AS-M and LH: initial draft; DH, EMM, MS-H, LH: critical revision, All authors: read and approved the final version.
Keywords: 25-hydroxyvitamin D, Covid-19, ethnicity, black people, inflammation.

\section{References}

1 Liu N et al (2021) Int J Infect Dis 104: 58-64.

2 O'Neill C (2016) et al J Steroid Biochem Mol Biol 173. 245-252.

3 ICNARC April 10th, (2020).

4 Kumar R et al (2021) Virus Research 292. 198235.

\section{$451 / 52$ \\ Effect of a Probiotic ${ }^{\circledR}$ on the Intestinal Immune System}

Tomás Cobos, L. ; Viadel Crespo, B. ${ }^{1}$; Sirvent Segura, E. ${ }^{2}$; Morant, L. ${ }^{2}$; Gallego Vendrell, $E .{ }^{1}$; Soriano-Romaní, L. ${ }^{1}$

${ }^{1}$ AINIA; ${ }^{2}$ Biopartner

The objective is to evaluate the integral effect of a probiotic ${ }^{\oplus}$ on the intestinal immune system: on the microbiota and on immune biomarkers.

Colonic microbiota is key for an adequate development of the intestinal mucosa and the immune system associated with it, so compounds that modulate the colonic microbiota are in turn modulating the immune response (1). In this sense, probiotic bacteria can act as immunomodulators, immune activators or immune suppressors, which have an appreciable and beneficial influence on homeostasis of the immune system (2).

The intestinal mucosa was reproduced by an in vitro integrated system. The colonic microbiota was reproduced by the in vitro digester for colonic fermentation and the intestinal epithelium by a cell model based on a co-culture of intestinal cells (Caco- 2 cell line) and macrophages cells (differentiated THP1 cell line). First, the impact of the probiotic on microbiota was monitoring on the microbial profile and its metabolites, analysing specific microbial species and its metabolites, the short chain fatty acids (SCFA). Later, the effect of the digested probiotic on cellular inflammatory biomarkers was evaluated analysing IL-6, COX-2 and NO.

The in vitro treatment of the probiotic $c^{\circledast}$ improved the microbiological profile and the microbial metabolism increasing the produced SCFAs. Moreover, the probiotic ${ }^{\circledR}$ increased the genic expression of IL- 6 and COX-2. Therefore, the probiotic ${ }^{\circledast}$ presented a potential immunostimulant effect.

Acknowledgments: This research was included in the project SATISFOOD: "Development and validation of satiating foods through the integration of pre and post ingestive signals (IDI 20150579)", which was founded by CDTI in the programme "Strategic Program of Business Research Consortiums (CIEN)".

Financial Support: This research was included in the project SATISFOOD: "Development and validation of satiating foods through the integration of pre and post ingestive signals (IDI 20150579)", which was founded by CDTI in the programme "Strategic Program of Business Research Consortiums (CIEN)".

Conflict of interest: The authors declare the following financial interests/personal relationships which may be considered as 
potential competing interests: The financial support of the project has been carried out by Biopartner as a research project to develop new functional ingredients.

\section{Authorship:}

- LM and ESS from Biopartner made the selection and preparation of the probiotic product.

- BVC, EGV, from AINIA carried out the in vitro studies of colonic fermentation with the Digester Dynamic of Colonic fermentation.

- LTC and LS-R, from AINIA carried out the in vitro studies of the digested probiotic on cell models.

Keywords: Probiotic, intestinal microbiota, immunostimulant.

\section{References}

1 Lazar V., Ditu L. M. Pircalabioru G. G., Gheorghe I., Curutiu C., Holban A. M., Chifiriuc, M. C. Aspects of gut microbiota and immune system interactions in infectious diseases, immunopathology, and cancer, Frontiers in Immunology. 2018: Vol. 9, 1830.

2 Llewellyn, A., \& Foey, A. (2017). Probiotic Modulation of Innate Cell Pathogen Sensing and Signaling Events. Nutrients, 9(10), 1156.

\section{$451 / 53$ \\ Changes in the Immune Composition of Breast Milk during the Transition Period \\ Rio Aige, K. '; Azagra Boronat, I. '; Selma Royo, M. ${ }^{2}$; Rodríguez Lagunas, M. ${ }^{1}$; Collado, M. ${ }^{2}$; Pérez Cano, F. ${ }^{1}$ \\ ${ }^{1}$ Dept. Biochemistry and Physiology, Faculty of Pharmacy and Food Science, University of Barcelona, Spain; ${ }^{2}$ Institute of Agrochemistry and Food Technology (IATA-CSIC), National Research Councl, Valencia, Spain}

Breast milk composition changes during the breastfeeding stages (colostrum, transitional and mature milk) $(1,2)$. Previous studies have described how the levels of most of the immune components of breast milk decrease from colostrum to mature milk (3-5). However, little is known about the changes of milk immunoglobulin (Ig) profile and cytokine (CK) levels within the transitional stage, which is considered from 3-5 days until up to 2 weeks after delivery. For that, the Ig profile (IgA, IgM, IgE, IgG, IgG1, IgG2, IgG3 and IgG4) and the CK levels (GM-CSF, IFN $\gamma$, IL-1 $\beta$, IL-2, IL-4, IL-5, IL-6, IL-9, IL-10, IL-12, IL-13, IL-17, IL-18, IL-21, IL-22, IL-23, IL-27, TNF- $\alpha$ ) were quantified and compared between two sampling days within the transitional stage (day 7 and 15 of breastfeeding) in a mother-infant birth cohort (MAMI, n= 75 ) in the Spanish-Mediterranean area by ELISA and Multiplex Bead Immunoassays. The results showed dynamic changes of these immune factors during this short period of time. A higher concentration of IgA, IgE, IgG2, IgM, IL-1 $\beta$, IL-9, IL-6, IL-10, IL-17 and IL-5 was found in $\mathrm{d} 7$ with respect to $\mathrm{d} 15$. On the contrary, IFN $\gamma$ and IL-18 increased from d7 to d15. The differences found here reinforce the idea that breast milk is a highly dynamic source of these immune factors, and particularly in this period.
These results highlight the importance of the milk collection timing in interventional and descriptive studies, as just few days lead to dramatic different levels of breast milk immune composition.

Acknowledgments: The authors would like to thank Jaume Comas, head of the Flow Cytometry Unit of the CCiT-UB and the families involved in the study.

Financial Support: This work was supported by the research grant from LaMarató-TV3 (DIM-2-ELI, ref. 2018-27/30-31). KRA holds a fellowship from the Spanish Ministry of Economy, Industry and Competitiveness (FPU19/05150).

Conflict of Interest: The authors declare no conflict of interest.

Authorship: Conceptualization, FPC and MC; methodology, MRL, KRA, IAB; writing-original draft preparation, FPC, MRL, KRA and IAB; writing - review and editing, MSR and MC; funding acquisition, FPC and MC. All authors have read and agreed to the manuscript.

Keywords: Breast milk, Transitional milk, Immunoglobulins, Cytokines, IgA.

\section{References}

1. Agarwal S, Karmaus W, Davis S, Gangur V. Immune markers in breast milk and fetal and maternal body fluids: A systematic review of perinatal concentrations. J Hum Lact. 2011;27(2):171-86.

2. Dawod B, Marshall JS. Cytokines and soluble receptors in breast milk as enhancers of oral tolerance development. Front Immunol. 2019; 10:00016.

3. Castellote C, Casillas R, Ramírez-Santana C, Pérez-Cano FJ, Castell M, Moretones MG, et al. Premature Delivery Influences the Immunological Composition of Colostrum and Transitional and Mature Human Milk. J Nutr. 2011;141(6):1181-7.

4. Trégoat V, Montagne P, Béné MC, Faure G. Increases of Iga milk concentrations correlate with IgA2 increment. J Clin Lab Anal. 2001; 15(2):55-8.

5. Frost BL, Jilling T, Lapin B, Maheshwari A, Caplan MS. Maternal breast milk transforming growth factor-beta and feeding intolerance in preterm infants. Pediatr Res. 2014;76(4):386-93.

\section{$451 / 54$ \\ Cocoa Modulates Mesenteric Lymph Nodes Composition and Function in Rats Submitted to Acute Running Exercise}

\author{
Ruiz-Iglesias, P. ; Massot-Cladera, M. ${ }^{1}$; Azagra-Boronat, I. ; \\ Franch Masferrer, Á. '; Pérez-Cano, F. ${ }^{1}$; Castell Escuer, M. ${ }^{1,2}$ \\ ${ }^{1}$ Section of Physiology, Faculty of Pharmacy and Food Science, \\ UB; INSA-UB; ${ }^{2}$ CIBEROBN
}

We aimed to evaluate the effect of cocoa and cocoa fibre intake on the mesenteric lymph nodes (MLN) functionality of rats submitted to high-intensity acute exercise, thus to assess their potential preventive effect on the exercise-induced gastrointestinal syndrome (1). For this purpose, female Wistar rats were distributed into three groups depending on the diet: standard, $10 \%$ cocoa or $5 \%$ cocoa fibre diet. After 4 weeks, half the rats of each diet 
performed an exhausting running test in a treadmill. MLN were collected after $16 \mathrm{~h}$ and lymphocytes were immediately isolated. Lymphocyte subsets and function, by means of their proliferation capacity and cytokine release after Concanavalin A stimulation, were assessed. A single bout of exhausting exercise decreased the proportion of TCD8+ cells whereas increased that of TCD4+ cells, NKT cells and $\mathrm{T} \gamma \delta$ cells in MLN. Both cocoa-enriched diets increased the percentage of $\mathrm{T} \gamma \delta$ cells in sedentary animals and prevented the increase of NKT cells induced by exercise. Cocoa fibre diet also prevented the changes induced by acute exercise on the TCD8+ and TCD4+ cell proportions. In addition, both acute exercise and cocoa consumption changed the cytokine release pattern without modifying the lymphocytes proliferation ability. Among other changes, acute exercise increased IL-10 secretion, whereas the cocoa intake decreased the IFN $\gamma / \mathrm{IL}-4$ ratio. Overall, our results indicate that cocoa can prevent some of the changes induced by a single bout of intense exercise on MLN lymphocytes which encourages further studies to ascertain its role as a preventive strategy in exercise-induced gastrointestinal syndrome.

Acknowledgments: The authors would like to thank Raquel Gómez-Bris, Mariona Camps-Bossacoma, María J. RodríguezLagunas, Marta Périz and Karla Rio-Aige for their help with the laboratory work.

Financial Support: This study was supported by the Spanish Ministry of Science and Innovation (AGL2016-76972-R, AEI/ FEDER, UE). P.R.-I. holds a grant from the Spanish Ministry of Education, Culture and Sport (FPU18-00807).

Conflict of Interest: None.

Authorship: MCE and FP-C. conceived and designed the experiments; P.R.-I., M.M.-C., I.A.-B. and AFM performed the experiments; P.R.-I. analysed the data and wrote the manuscript. All authors have read, reviewed and approved the final version of the manuscript.

Keywords: Cocoa; fibre; intestinal immunity; cytokines; acute exercise.

\section{Reference}

1 Costa RJS, Snipe RMJ, Kitic CM, Gibson PR. Systematic review: exerciseinduced gastrointestinal syndrome-implications for health and intestinal disease. Aliment Pharmacol Ther. 2017;46(3):246-65.

\section{$451 / 55$ \\ Influence of the Consumption of Peruvian Cocoa Populations on a Rat Model of Allergic Asthma}

\author{
Massot-Cladera, M. ${ }^{1}$; Périz, M. ${ }^{1}$; Pastor-Soplin, S. ${ }^{2}$; \\ Castell Escuer, M. ${ }^{1}$; Best, I. ${ }^{2}$; Pérez-Cano, F. ${ }^{1}$ \\ ${ }^{1}$ Physiology Section, Faculty of Pharmacy and Food Science, \\ University of Barcelona, INSA-UB, Spain; ${ }^{2}$ Programa Cacao, \\ Ingeniería Agroforestal, Facultad de Ciencias Ambientales, \\ UCSUR, Lima, Peru
}

The study aimed to investigate the influence of two Peruvian cocoa populations with different content of bioactive components
(1) on the immune response developed in a allergic asthma rat model. For this purpose, Brown Norway rats were fed a standard diet or diets containing 10\% cocoa paste either from 'Amazonas Peru' (APC) or 'Criollo de Montaña' (CMC) Peruvian populations (fine or flavour cocoa) or an ordinary cocoa (OC). After one-week diet, asthma was induced by two intraperitoneal sensitizations and an intranasal challenge with ovalbumin (OVA). Serum specific IgE, IgM, IgG1, IgG2a, IgG2b and IgG2c antibodies were quantified $24 \mathrm{~h}$ after intranasal challenge. Phenotype of cells contained in the bronchoalveolar lavage fluid (BALF) was determined by flow cytometry. The allergic asthma induced the production of serum specific antibodies whereas the three cocoa diets partially prevented it. In particular, anti-OVA IgG1 levels after OC, APC and $\mathrm{CMC}$ diets were lower than those after the standard diet, whereas cocoa diets induced a trend to lower anti-OVA IgG2a (related to Th2 response). Anti-OVA IgE synthesis was mainly prevented by the OC diet. Anti-OVA IgG2b and IgG2c (associated with Th1 response) and IgM levels were not modified by the cocoa diets. The BALF eosinophil counts increased in standard diet-fed asthmatic animals but did not in those consuming any cocoa diet. In conclusion, the consumption of Peruvian cocoa populations was able to modulate immune response in a rat model of allergic asthma differently according to the origin and content of bioactive compounds of these cocoa populations.

Acknowledgments: The authors thank Nerea Moreno Pena and Anna Cutrina for their technical assistance.

Financial Support: The present work was funded by the National Fund for Scientific, Technological and Technological Innovation Development (FONDECYT) of the National Council of Science, Technology and Technological Innovation (CONCYTEC), Contract 137-2017-FONDECYT.

Conflict of Interest: The authors declare no conflict of interest

Authorship: All authors designed the experiments; M.P and M.M.-C. carried out them; M.M-C and MCE analysed the data and wrote the abstract and M.P., S.P.-S., I.B., and FP-C. reviewed it.

Keywords: Allergic asthma, Th2 immune response, Peruvian cocoa

\section{Reference}

Périz M, Pérez-Cano FJ, Cambras T, Franch À, Best I, Pastor-Soplin S, et al. Attenuating effect of Peruvian cocoa populations on the acute asthmatic response in Brown Norway rats. Nutrients. 2020 Aug;12(8):1-21. 


\section{$451 / 56$}

\section{Changes in Peyers' Patches and Intestinal IgA in Acute-Exercised Rats Consuming a Cocoa Diet}

Ruiz-Iglesias, P. ${ }^{1}$; Périz, M. ${ }^{1}$; Rio-Aige, K. ${ }^{1}$; Rodríguez-Lagunas, M. ${ }^{1}$; Castell Escuer, M. ${ }^{1,2}$; Pérez-Cano, F. ${ }^{1}$

${ }^{1}$ Secció de Fisiologia, Facultat de Farmàcia, Universitat de Barcelona (UB); INSA-UB; ${ }^{2}$ Secció de Fisiologia, Facultat de Farmàcia, Universitat de Barcelona (UB); INSA-UB; CIBEROBN

The aim of the current study was to evaluate the impact of cocoa intake on intestinal IgA and Peyer's patches (PP) lymphocytes of rats following an acute exercise, as well as to elucidate the involvement of cocoa fibre content in such effects. Female Wistar rats were fed either a standard diet, a diet containing $5 \%$ cocoa fibre or a diet containing $10 \%$ cocoa for 4 -weeks. At the end of the study, half the rats performed an exhausting running test in a treadmill. Faeces made during the exhaustion test were weighted and processed for water content assessment. Faecal samples were also collected at the beginning and at the end of the study for IgA quantification. PP were obtained $16 \mathrm{~h}$ after the exhaustion test and the proportion of lymphocyte populations and subpopulations were determined by flow cytometry. Cocoa fibre prevented the increase in intestinal motility induced by acute exercise, as shown by a decreased total faecal mass and water content. A lower faecal IgA content was observed in all the dietary groups after exercise. Concerning the PP lymphocyte composition, cocoa intake increased the percentage of NKT and T $\gamma \delta$ cells in sedentary animals, modifying the proportion of the $\mathrm{T} \gamma \delta \mathrm{CD} 8 \alpha \alpha+$ and CD $8 \alpha \beta+$ subsets, while it decreased the T CD4+/T CD8+ cell ratio in both runner and sedentary rats. Overall, cocoa, by means of its fibre content, may prevent the alterations in gut motility induced by intensive exercise and modify the small intestine lymphoid tissue composition.

Acknowledgments: The authors would like to thank Raquel Gómez-Bris, Mariona Camps-Bossacoma and Malén MassotCladera for their help with the laboratory work.

Financial Support: This study was supported by the Spanish Ministry of Science and Innovation (AGL2016-76972-R, AEI/ FEDER, UE). P.R.-I. holds a grant from the Spanish Ministry of Education, Culture and Sport (FPU18-00807).

Conflict of Interest: None.

Authorship: MCE and FP-C conceived and designed the experiments; P.R.-I., M.P., K.R.-A. and MR-L. performed the experiments; P.R.-I. analysed the data and wrote the manuscript.

Keywords: Fibre, intestinal immunity, acute exercise, peyer patches, IgA.

\section{References}

\section{$451 / 57$ \\ Impact of Diets Rich in Omega 6 Fatty Acids on Thymus of Growing Rats}

Slobodianik, N.; Perris, P.; Mambrin, M.; Fernandez, I.; Feliu, M.

University of Buenos Aires. Faculty of Pharmacy and

Biochemistry, Nutrition

Dietary lipids have an important role to regulate immune system. Thymus shows important functional changes in response to nutritional disorders $(1,2)$. The aim of this work is to analyze the effect of diets rich in omega 6 fatty acids in normal and high concentration on thymus' fatty acid profiles of growing rats. Weanling Wistar rats were fed during 10 (10D) and 40 (40D) days with 15\% and $42 \%(\mathrm{~F} \%=\mathrm{Kcal}$ lipids $/ 100$ total $\mathrm{Kcal})$; dietary fat was provided by sunflower oil (S15 and S42 groups). Control group received diet (soy oil, F\%=15) according to AIN'93. Thymus fatty acid profiles were determined by gas chromatography. The statistical analysis used analysis of variance (ANOVA) and Dunnett as post test $\left({ }^{*} \mathrm{p}<0.01\right)$. Results: Were $(\%$ Area $)$ : Thymus fatty acid profile: OLEIC F15-10D = 13.50 $\pm 2.11 ;$ F42-10D = 19.17 \pm 1.37 ; CONTROL $10 \mathrm{D}=18.22 \pm 3.23 ; \mathrm{F} 15-40 \mathrm{D}=16.13 \pm 2.72 ; \mathrm{F} 42-40 \mathrm{D}=14.91 \pm 1.90$; CONTROL 40D = 16.84 \pm 5.20 ; LINOLEIC F15-10D = 10.46 \pm 3.34 ; $\mathrm{F} 42-10 \mathrm{D}=15.19 \pm 1.10 ; \mathrm{CONTROL} 10 \mathrm{D}=10.26 \pm 1.37 ; \mathrm{F} 15-40 \mathrm{D}=$ 9.94 $\pm 3.61 ; \mathrm{F} 42-40 \mathrm{D}=29.49 \pm 1.26^{*} ; \mathrm{CONTROL} 40 \mathrm{D}=10.22 \pm 3.10$ alfa-LINOLENIC F15-10D $=0.32 \pm 0.03^{*}$; F42-10D = 0.29 $\pm 0.07^{*}$; CONTROL $10 \mathrm{D}=0.59 \pm 0.09 ; \mathrm{F} 15-40 \mathrm{D}=0.26 \pm 0.03^{*} ; \mathrm{F} 42-40 \mathrm{D}=$ $0.26 \pm 0.03^{*}$; CONTROL $40 \mathrm{D}=0.75 \pm 0.11$; EPA F15-10D $=$ $0.17 \pm 0.08$; F42-10D $=0.24 \pm 0.12$; CONTROL $10 \mathrm{D}=0.50 \pm 0.12$; $\mathrm{F} 15-40 \mathrm{D}=0.15 \pm 0.07 ; \mathrm{F} 42-40 \mathrm{D}=0.14 \pm 0.05$; CONTROL $40 \mathrm{D}=$ $0.15 \pm 0.07$ DHA F15-10D $=0.39 \pm 0.10 ;$ F42-10D $=0.31 \pm 0.08$; CONTROL $10 \mathrm{D}=0.52 \pm 0.16 ; \mathrm{F} 15-40 \mathrm{D}<0.05^{\star} ; \mathrm{F} 42-40 \mathrm{D}<0.05^{\star}$; CONTROL $40 \mathrm{D}=0,52 \pm 0,14$. The results observed demonstrate a decrease on the levels of alfa linolenic acid, independent of F\%. F15D40 and F4240D groups showed lower DHA. F42D40 group presents high levels of linoleic acid due sunflowers oil contains high level of linoleic acid. The results suggest that it was exacerbated $\omega 6$ family and decreased $\omega 3$ family. Thymus, characterized by a high rate of celular turnover, is severely affected by nutritional disorders and particularly by distortion in the proportion and type of fat.

Acknowledgments: University of Buenos Aires.

Financial Support: Supported by UBACyT 20020190100093BA.

Conflict of Interest: There is no conflict of interest.

Authorship: All authors contributed equally.

Keywords: Thymus-, Fatty, acids-, diet-, rat.

\section{References}

1 Inmunonutrición en la salud y la enfermedad, segunda edición. A. Marcos ed. Panamericana. Madrid. 2019.

2 Innes J, Calder P. Omega-6 fatty acids and inflammation. Prostag Leukotr Ess 2018; 132: 41-48. 
$451 / 58$

Effect of Different In Vitro Stimulation Levels of a Tomato Extract on Cytokine Production

Godoy, M. ${ }^{1}$; Díaz, L. ${ }^{2}$; Nova, E. ${ }^{2}$; Insani, E. ${ }^{3}$; Slobodianik, N. ${ }^{4}$; $\operatorname{Marcos}, A^{2}$

${ }^{1}$ INTA, Food Technology Institute. UBA, Faculty of Pharmacy and Biochemistry, Buenos Aires, Argentina; ${ }^{2}$ mmunonutrition Group, ICTAN, CSIC, Madrid, Spain; ${ }^{3}$ INTA. Food Technology Institute, Buenos Aires, Argentina; ${ }^{4}$ University of Buenos Aires. Faculty of Pharmacy and Biochemistry, Nutrition. Buenos Aires, Argentina

Objective: This study was aimed to evaluate the in vitro effect of a lipophilic tomato extract (TE) addition on cytokine production by peripheral blood mononuclear cells (PBMCs).

Methods: Human PBMCs ( $\mathrm{n}=7$, women's independent samples) were isolated and cultured with PHA/LPS for $48 \mathrm{~h}$ to stimulate cytokine production. Five groups were considered: 1) PBMCs + PHA/LPS + $1 \mu \mathrm{M}$ of $\alpha$-tocopherol from TE (PMBC-1), 2) PBMCs + PHA/LPS + $10 \mu \mathrm{M}$ of $\alpha$-tocopherol from TE 10 (PBMC-10), 3) PBMCs + PHA/LPS $+20 \mu \mathrm{M}$ of $\alpha$-tocopherol from TE (PBMC$20)$, 4) PBMCs+PHA/LPS, as the stimulation control group (C), and 5) PBMCs without PHA/LPS, as the basal group (B). TNFa, IL- $1 \beta$, IFN- $\gamma$, IL-2, IL-4, IL-13, IL-17A and IL-10 were measured in culture supernatants (MILLIPLEX MAP- HCYTOMAG-60K, LuminexMAGPIX System). Statistical ANOVA analysis and Pearson correlations were assessed.

Results: TE attenuated PBMC response to PHA/LPS in PBMC20 for IFN- $\gamma$, IL-17A and IL-10 and exacerbated PBMC response to PHA/LPS in PBMC-10 for IL-4, IL-13 and IL- $1 \beta$ in comparison with $C(p \leq 0.05)$. IL-10 and IL- $1 \beta$ levels in B were positively correlated with $\mathrm{C}$ and with increasing amounts of PBMC-1, PBMC-10 and PBMC-20. All Pearson correlations were above 0.9453 ( $\mathrm{p} \leq$ 0.0013).

Conclusions: The lipophilic tomato extract affects PBMCs response to mitogens in a dose-dependent manner depending on the cytokine assessed. PBMC of individuals who presented lower IL-10 and IL- $1 \beta$ basal levels also showed lower levels after stimulation suggesting that this response is dependent on the level of these cytokines prior to stimulation.

Acknowledgments: We are grateful to María Luján Calcagno from University of Buenos Aires. Faculty of Pharmacy and Biochemistry, Mathematics. Buenos Aires, Argentina for her valuable statistical analysis assistance and the PBMC donors.

Financial Support: This work was supported by the Intramural Project, CSIC (grant number 201270E121) and the Postgraduate and Advanced Training Programs, INTA (MFG, grant number 1177/14).

Conflict of Interest: None.

Authorship: All authors participated in the design and development of the project, as well as in the analysis of data and writing of the abstract.

Keywords: Tomato extract, cytokines, PBMCs.

\section{References}

Belisle SE, Leka LS, Dallal GE, Jacques PF, Delgado-Lista J, Ordovas JM, Meydani SN. Cytokine response to vitamin E supplementation is dependent on pre-supplementation cytokine levels. Biofactors 2008; 33(3): 191- 200.

Mills LM, Wilson H, Thies F. Lycopene inhibits lymphocyte proliferation through mechanisms dependent on early cell activation. Mol. Nutr. Food Res. 2012; 56: 1034-1042.

\section{$451 / 59$ \\ Table Olives Modify Fecal Microbiota in Spontaneously Hypertensive Rats and their Normotensive Controls}

Gómez Contreras, A. ' ; Franco-Ávila, T. ${ }^{1}$; Miró, L. ' ; Juan, M. ${ }^{1}$; Moretó, M. ${ }^{1}$; Planas, J. ${ }^{1}$; Márquez Contreras, E. ${ }^{2}$

${ }^{1}$ Departament de Bioquímica i Fisiología, Facultat de Farmàcia i Ciències de l'Alimentació, UB. ${ }^{2}$

The fruit of Olea europaea L. contains bioactive compounds with health protecting activities that may involve microbiota modulation. We have analyzed the effects of Arbequina olives consumption on fecal microbiota in spontaneously hypertensive (SHR) and in Wistar-Kyoto normotensive (WKY) rats. Treated groups were administered a homogeneous suspension $(10 \mathrm{~mL} / \mathrm{kg})$ of the edible part of Arbequina table olives $(3.85 \mathrm{~g} / \mathrm{kg}$; suspended in water) at 14 weeks of age for 49 days. The composition of the microbiome was analyzed by sequencing fecal $16 \mathrm{~S}$ rRNA genes using the Illumina MiSeq platform (1). At 14 weeks of age, $\alpha$ - and $\beta$-diversity were similar in both strains, and no differences were observed in the abundance of Firmicutes, Bacteroidetes and Actinobacteria, nor in the Firmicutes-Bacteroidetes (F/B) ratio. At 21 weeks, bacterial diversity was unchanged in the control groups. However, higher abundance of Firmicutes $(\mathrm{p}=0.011)$ and lower abundance of Bacteroidetes were observed in SHR ( $\mathrm{p}=0.003)$ compared to $\mathrm{WKY}$ rats, resulting in a higher $\mathrm{F} / \mathrm{B}$ ratio $(\mathrm{p}=0.023)$. Moreover, genus Phascolarctobacterium, Parabacteroides and Prevotella were more abundant in WKY $(\mathrm{p}<0.05)$. The ingestion of table olives reduced the F/B ratio in SHR, it stimulated the growth of $L$. acidophilus, L. crispatus and B. choerinum $(\mathrm{p}<0.05)$ and reduced the abundance of Anaerotruncus ( $\mathrm{p}=0.012)$ in the treated groups. In addition, the treatment promoted the growth of $B$. thermacidophilum and $B$. animalis $(\mathrm{p}<0.05)$ in SHR, while the abundance of Prevotella $(\mathrm{p}=0.007)$ decreased in WKY. In conclusion, consumption of Arbequina table olives reduced the $\mathrm{F} / \mathrm{B}$ ratio in SHR and favored the growth of health-promoting bacteria.

Acknowledgments: The authors thank the Unitat d'Experimentació Animal de Farmàcia (CCiTUB).

Financial Support: This work was funded by grants AGL201341188 from Ministerio de Economía y Competitividad and 2017SGR945 from Generalitat de Catalunya, Spain. AGC holds a pre-doctoral fellowship from PRONABEC, Ministerio de Educación, Perú.

Conflict of Interest: The authors declare no conflict of interest. 
Authorship: JM and MM conceived and designed the experiments. AGC, TF-A, MJ and LM performed the experiments. AGC, JM and MM analyzed the data and wrote the abstract. All authors read and approved the final communication.

Keywords: Arbequin, gut microbiota, hypertension, rat.

\section{Reference}

1 Moretó M, Miró L, Amat C et al. (2020) Dietary supplementation with spray-dried porcine plasma has prebiotic effects on gut microbiota in mice. Sci Rep 10, 2926.

$451 / 60$

\section{Impact of Iron Deficiency Anemia on Cecum Microbiota in Animal Model}

Díaz Prieto, L.; Redondo Useros, N.; Nova, E.; Gonzalez Zancada, N.; Gómez Martínez, S.; Marcos, A.

Immunonutrition group. Department of Nutrition and Metabolism (DMN). Institute of Food Science, Technology and Nutrition (ICTAN), Madrid, Spain

The aim of this study was to evaluate the changes produced in the cecum microbiota in an animal model of chronic iron deficiency anemia. Four week-old male C57BL6/J mice $(n=20)$, were fed ad libitum a control diet or an iron-deficient diet (50 or $4 \mathrm{mg}$ of iron/kg chow, respectively) for 8 weeks. An automatic hematological counter was used to obtain red cell line analysis. Transferrin levels were determined by Abbott ARCHITECT multi-analyte autoanalyzer. The gut microbiota was analyzed in cecum samples by qPCR for quantification of group-specific 16S rRNA genes. Differences between groups were analyzed by independent samples T test, and variable associations by Pearson correlation. The iron deficiency anemia (IDA) group showed higher levels of Akkermansia muciniphila and Enterococcus spp. $(\mathrm{p}<0.01)$ compared to the control group, which were also negatively correlated to red blood cells, hemoglobin, hematocrit and mean corpuscular volume. In addition, the levels of Bacteroides, Blautia cocoidesEubacterium rectale and Clostridium cluster IV were lower in the IDA-group $(p<0.05)$ and positively correlated to the erythrocyte indices above mentioned. In addition, transferrin levels were higher in the IDA group and positively correlated to Akkermansia muciniphila and Enterococus spp. $(\mathrm{p}<0.01)$ and negatively to Clostridium cluster IV $(\mathrm{p}<0.05)$. Conclusion: The relationship between erythrocyte levels and specific bacterial groups, as well as the bacterial dysbiosis induced by iron deficiency suggests the need of further follow-up assessments to evaluate the evolution of dysbiosis and to consider the therapies based on iron supplements (1).

Acknowledgments: To all the staff of the Animal Facility of the Center of Biological Research (CIB), Spanish National Research Council (CSIC).

Financial Support: ITF RESEARCH PHARMA, S.L.U.

Conflict of Interest: The authors declare no conflict of interest.
Authorship: LDP, NRU, NGZ and SGM performed analytical techniques, AM, EN and LDP designed the study.

Keywords: Iron deficiency, gut microbiota, erythrocytes, dysbiosis.

\section{Reference}

1 Muleviciene A, D’Amico F, Turroni S, Candela M, Jankauskiene A. Iron deficiency anemia-related gut microbiota dysbiosis in infants and young children: A pilot study. Acta Microbiologica et Immunologica Hungarica. 2018 Dec;65(4):551-564. DOI: 10.1556/030.65.2018.045

\section{$451 / 61$ \\ The Relationship of Lifestyle Determinants with Immunological and Hormonal Biomarkers in Adolescents with Down syndrome. The UP\&DOWN Study}

Gutierrez-Hervas, A. ' ; Gómez-Martínez, S. ${ }^{2}$ Izquierdo-Gómez, R. ${ }^{3}$; Veiga, O. ${ }^{4}$; Marcos, A. ${ }^{2}$

${ }^{1}$ Institute of Food Science, Technology and Nutrition and Nursing Department, University of Alicante; ${ }^{2}$ Institute of Food Science, Technology and Nutrition (ICTAN). Spanish National Research Council (CSIC); ${ }^{3}$ GALENO Research Group and INiBICA. Cádiz, Spain; ${ }^{4}$ Department of Physical Education, Sport and Human Movement, University Autonomous of Madrid

The objective of this study was to evaluate the relationships between several inflammatory biomarkers (insulin, leptin, adiponectin, galactin-3, total proteins, interleukin-6 [IL-6], C-reactive protein $[\mathrm{CRP}]$ and tumor necrosis factor [TNF- $\alpha$ ] and lifestyle determinants in a sample of adolescents with Down syndrome (DS). Hundred and one DS adolescents (aged 10-20) were recruited for the UP\&DOWN Study. Sleep time, screen time, moderate-vigorous physical activity (MVPA) (measured by accelerometry), adherence to the Mediterranean diet, height and waist were measured. Waist-to-height ratio (WHtR), as abdominal obesity index, and (MVPA), according to Helena study cut-points criteria, were calculated. Dichotomized variables: quality of the Mediterranean diet (adequate $\geq 8$ points $^{1}$; inadequate $<8$ points), sleep time (adequate $8-10$ hours/day ${ }^{2}$; inadequate $<8$ or $>10$ hours/day), screen time (adequate $<2$ hours/day ${ }^{3}$; inadequate $\geq 2$ hours/day), MVPA (adequate $\geq 60$ minutes/day ${ }^{4}$; inadequate $<60$ minutes/day) and abdominal obesity (when WHtR $\geq 0.51$ in boys and $\geq 0.50$ in girls ${ }^{5}$; or not). Student's t test was performed. Higher insulin and total proteins levels as well as a trend to be higher galactin-3 levels were found in DS adolescents who did not sleep adequate time. Higher levels of galactin-3 and total proteins levels were shown in adolescents who did not fit with MVPA guidelines. Insulin and leptin levels were higher in adolescents with abdominal obesity. No associations were found in IL-6, CRP, TFN- $\alpha$ and adiponectin levels. These results highlight that sleep time, MVPA and abdominal obesity are important factors to bear in mind when evaluating the nutritional and hormonal status of adolescents with DS. 
Acknowledgments: To the adolescents who participated in this study, as well as to their parents and families.

Financial Support: DEP 2010-21662-C04-00 grants from the National Plan for Research, Development and Innovation $(\mathrm{R}+\mathrm{D}+\mathrm{i})$ MICINN.

Conflict of Interest: The authors declare no conflict of interests.

Authorship: AG-H: analysing the data and writing the document.

SG-M: carrying out the study and writing the document.

RI-G: carrying out the study and reviewing the document.

OV: reviewing the document.

AM: writing and reviewing the document.

Keywords: Hormonal biomarkers; lifestyles; adolescents; Down syndrome.

\section{References}

1 Serra L, Ribas L, Ngo J, Ortega RM, Pérez C, Aranceta J. Alimentación, jóvenes y dieta mediterránea en España. Desarrollo del KIDMED, índice de calidad de la dieta mediterránea en la infancia y la adolescencia. En: Alimentación infantil y juvenil: Estudio enKid (1998-2000). Barcelona: Masson; 2004. p. 51-9.

2 Paruthi S, Brooks LJ, D'Ambrosio C, Hall WA, Kotagal S, Lloyd RM, Malow BA, Maski K, Nichols C, Quan SF, Rosen CL, Troester MM, Wise MS. Consensus statement of the American Academy of Sleep Medicine on the recommended amount of sleep for healthy children: methodology and discussion. J Clin Sleep Med 2016;12(11):1549-1561.

3 Council on communications and media. Children, adolescents, and the media. Pediatrics. 2013;132(5):958-961. DOI: 10.1542/peds.2013-2656. PMID: 28448255.

4 European Commission. EU physical activity guidelines: Recommended policy actions in support of health-enhancing physical activity. Available at: http://ec.europa.eu/sport/library/policy documents/euphysical-activity-guidelines-2008 en.pdf.

5 Marrodan MD, Roman J, González-Montero M, López-Ejeda N, Cabañas MD, Prado C. Precisión diagnóstica del índice cintura-talla para la identificación del sobrepeso y de la obesidad infantil. Med Clin (Barc). 2013;140(7):296-301.

\section{$451 / 62$}

\section{The Influence of Lifestyle Determinants on Biochemical Biomarkers in Adolescents with Down Syndrome. The UP\&DOWN Study}

\author{
Gómez-Martínez, S. '; Gutierrez-Hervas, A. 2'; Izquierdo-Gómez, R. ${ }^{3}$; \\ Veiga, O. ${ }^{4}$; Marcos, $A .^{1}$ \\ ${ }^{1}$ Institute of Food Science, Technology and Nutrition (ICTAN). \\ Spanish National Research Council (CSIC); ${ }^{2}$ Institute of Food \\ Science, Technology and Nutrition and Nursing Department, \\ University of Alicante; ${ }^{3}$ GALENO Research Group and INiBICA. \\ Cádiz, Spain; ${ }^{4}$ Department of Physical Education, Sport and \\ Human Movement, University Autonomous of Madrid
}

This study was aimed to evaluate the influence of lifestyle determinants on several biochemical marker levels related to cardiovascular disease risk, such as complement factors (C3 and C4), glucose, total cholesterol and fractions (HDL-cholesterol and LDL-cholesterol), and triglycerides in a sample of adolescents with Down syndrome (DS). A group of 101 DS adolescents (aged 10-20) was recruited for the UP\&DOWN Study. Sleep time, screen time, moderate-vigorous physical activity (MVPA) (measured by accelerometry), the adherence to the Mediterranean diet, height and waist were measured. Both waist-to-height ratio (WHtR), as abdominal obesity marker, and MVPA, using the Helena study cut points criteria, were calculated. Dichotomized variables: quality of Mediterranean diet (adequate $\geq 8$ points $^{1}$; inadequate $<8$ points), sleep time (adequate $8-10$ hours/ $\mathrm{day}^{2}$; inadequate $<8$ or $>10$ hours/ day), screen time (adequate $<2$ hours $/ \mathrm{day}^{3}$; inadequate $\geq 2$ hours/ day), MVPA (adequate $\geq 60$ minutes $/ \mathrm{day}^{4}$; inadequate $<60 \mathrm{~min}$ utes/day) and abdominal obesity (when WHtR $\geq 0.51$ in boys and $\geq 0.50$ in girls ${ }^{5}$; or not). Student's t test was used. None of the lifestyle determinants evaluated showed an influence on glucose and HDL-cholesterol levels. However, triglyceride and total cholesterol levels tended to be higher among DS adolescents who exceeded screen time recommendations. Moreover, these both biomarkers together with LDL-cholesterol were shown higher among those DS adolescents who do not fit MVPA guidelines. Likewise, adolescents with abdominal obesity showed higher C3 and $\mathrm{C} 4$ component factors as well as triglycerides and total cholesterol levels. Therefore, the influence of physical activity and abdominal obesity on these biochemical biomarkers should be strongly highlighted in this population.

Acknowledgments: The authors would like to acknowledge the involvement of the child and youth population who participated in this study, as well as to their parents and families.

Financial Support: DEP 2010-21662-C04-00 grants from the National Plan for Research, Development and Innovation $(\mathrm{R}+\mathrm{D}+\mathrm{i})$ MICINN.

Conflict of Interest: The authors declare no conflict of interests.

Authorship: AG-H: analysing the data and writing the document.

SG-M: carrying out the study and writing the document.

RI-G: carrying out the study and reviewing the document.

$\mathrm{OV}$ : reviewing the document.

AM: writing and reviewing the document.

Keywords: Biochemical-biomarkers; lifestyles; adolescents; Down syndrome.

\section{References}

1 Serra L, Ribas L, Ngo J, Ortega RM, Pérez C, Aranceta J. Alimentación, jóvenes y dieta mediterránea en España. Desarrollo del KIDMED, índice de calidad de la dieta mediterránea en la infancia y la adolescencia. En: Alimentación infantil y juvenil: Estudio enKid (1998-2000). Barcelona: Masson; 2004. p. 51-9.

2 Paruthi S, Brooks LJ, D’Ambrosio C, Hall WA, Kotagal S, Lloyd RM, Malow BA, Maski K, Nichols C, Quan SF, Rosen CL, Troester MM, Wise MS. Consensus statement of the American Academy of Sleep Medicine on the recommended amount of sleep for healthy children: methodology and discussion. J Clin Sleep Med 2016;12(11):1549-1561.

3 Council on communications and media. Children, adolescents, and the media. Pediatrics. 2013;132(5):958-961. DOI: 10.1542/peds.2013-2656. PMID: 28448255. 
4 European Commission. EU physical activity guidelines: Recommended policy actions in support of health-enhancing physical activity. Available at: http://ec.europa.eu/sport/library/policy documents/euphysical-activity-guidelines-2008 en.pdf.

5 Marrodan MD, Roman J, González-Montero M, López-Ejeda N, Cabañas MD, Prado C. Precisión diagnóstica del índice cintura-talla para la identificación del sobrepeso y de la obesidad infantil. Med Clin (Barc). 2013;140(7):296-301

\section{$451 / 63$}

\section{Changes in the Immunoglobulin Profile of Lactating Rats Supplemented with Extra Virgin Olive Oil}

Grases-Pintó, B. ${ }^{1}$; Zhan-Dai, S. ; Morales-Ferré, C. ' Pérez-Cano, F. ${ }^{1}$; Vallverdú-Queralt, $A^{2}{ }^{2}$; Rodríguez-Lagunas, $M .{ }^{1}$

${ }^{1}$ Dept. Biochemistry and Physiology; Faculty of Pharmacy and Food Science, University of Barcelona, Spain; ${ }^{2}$ Dept. Nutrition, Food Science and Gastronomy; Faculty of Pharmacy and Food Science, University of Barcelona, Spain; ${ }^{3}$ Eliminate affiliation 3

Extra virgin olive oil (EVOO), a key component of the Mediterranean diet, has health-promoting properties as a result of its high content of polyphenols (1). However, little is known about the effect of EVOO on the immune system and even less during pregnancy and lactation periods. The objective of this study was to assess the impact of EVOO supplementation on the rat immune system during pregnancy and lactation. For that, the immunoglobulin (Ig) levels of different lymphoid tissues, plasma and breast milk of dams supplemented orally with $10 \mathrm{~mL} / \mathrm{Kg}$ of EVOO were quantified by ELISA and Multiplex Bead Immunoassays. A refined oil at the same dosage was used as control oil. No differences between groups in the Ig profile were found before pregnancy. An increase in IgG2b and IgG2c levels (subclasses associated to Th1 response) were observed in the plasma of animals receiving EVOO at day 14 . The pregnancy induced a decrease in the Th1/Th2 ratio of plasmatic Ig that continued diminishing until the end of the lactation. None of the supplementations affected that pattern. Although EVOO supplementation induced lower plasma levels of IgA and IgM at the end of lactation compared to the control oil, the animals from EVOO group had higher IgA in milk and in mammary gland homogenates. Moreover, an increase in IgM levels in the mesenteric lymph nodes was also observed in EVOO group. Overall, the supplementation with EVOO induced changes in the Ig profile of dams by promoting higher levels at mucosal sites.

Acknowledgments: The authors would like to thank Jaume Comas, head of the Flow Cytometry Unit of the CCiT-UB and Ksenia Morkuntsova for her technical support.

Financial Support: This work was supported by the research grant from the INSA-UB (FRI2019).

Conflict of Interest: The authors declare no conflict of interest.

Authorship: Conceptualization, MR-L and A.V-Q; methodology, MR-L, B.G-P, C.M-F, and S.Z-D; writing-original draft preparation, FP-C, MR-L, and B.G-P; writing-review and editing, FP-C, MR-L and A.V-Q; funding acquisition, MR-L and A.VQ. All authors have read and agreed to the manuscript.
Keywords: Extra virgin olive oil, Breast milk, Immunoglobulins, lymphoid tissues.

\section{Reference}

1 Yubero-Serrano EM, Lopez-Moreno J, Gomez-Delgado F, Lopez-Miranda J. Extra virgin olive oil: More than a healthy fat. Vol. 72, European Journal of Clinical Nutrition. Springer Nature; 2019. p. 8-17.

\section{$451 / 64$ \\ Effect of Moringa Oleifera Leaf on Intestinal Microbiota Composition of Prediabetic Adult Subjects}

Nova, E. ; Díaz, L. ' ; Gómez-Martínez, S. '; González-Romero, E. '; Calle, N. ${ }^{3}$; Marcos, $A .^{1}$

${ }^{1}$ Institute of Food Science and Technology and Nutrition (ICTAN)-CSIC; ${ }^{2}$ C.S. Cea Bermúdez, Servicio Madrileño de Salud;

${ }^{3}$ Madrid-Salud, Ayuntamiento de Madrid

The aim of this work was to assess whether the leaves of the Moringa oleifera (MO) plant change the intestinal microbiota composition during an intervention study performed in prediabetic adults. A double-blind placebo-controlled, parallel group intervention was conducted. Sixty five prediabetic subjects aged between 40 and 75 years finished the study and were included in this analysis (55\% females). Subjects under pharmacological treatment or suffering any serious disease were excluded. Participating subject consumed 6 daily capsules of MO dry leaves powder (400 $\mathrm{mg}$ /unit) (MO group, $\mathrm{N}=31$ ) or 6 placebo capsules (PLC group, $\mathrm{N}=34$ ) during 12 weeks. Results: No significant interaction "treatment*time" was found for any of the nine representative bacterial groups or species analyzed using qPCR. No differences were found between treatment groups neither before (T0) or after the intervention (T12). Since a significant decrease was observed in glycated hemoglobin (HbA1C) values in the MO group (T12: $5.79 \pm 0.37 \%$ vs. T0: $5.88 \pm 0.31 \%$; T test, $\mathrm{P}=0.025)(1)$, we analyzed the difference in basal bacterial group's abundance between subject improving and not improving HbAlC during the intervention. In the $\mathrm{MO}$ group, significantly lower basal Bacteroides abundance was found in subjects improving $\mathrm{HbAlC}$ during the intervention $(\mathrm{N}=18)$ compared to subjects whose HbA1C levels did not improve $(\mathrm{N}=13 ; \mathrm{P}=0.058)(9.45 \pm 0.53$ vs. $9.79 \pm 0.37 \mathrm{CFU}$ (Log)/g feces). However, In the PLC group no differences were found in bacterial group's abundances between subjects improving $(\mathrm{N}=13)$ and not improving $(\mathrm{N}=21) \mathrm{HbA} 1 \mathrm{C}$. Conclusion: Bacteroides abundance in basal microbiota might influence the beneficial effect of MO on glycemic control.

Acknowledgments: The authors would like to acknowledge the involvement of volunteers who participated in this study.

Financial Support: Ministerio de Economía, Industria y Competitividad (MINECO), Agencia Estatal de Investigación (AEI) and Fondo Europeo de Desarrollo Regional (FEDER, UE) (ref. AGL2017-86044-C2-1-R.).

Conflict of Interest: None. 
Authorship: LD, SG-M and EN performed analytical techniques, EGR and NC recruited patients, AM and EN designed the study.

Keywords: Moringa oleifera, dietary supplement, prediabetes, intestinal microbiota.

\section{Reference}

1 Vicente I. et al. Effect of Moringa oleifera as a dietary supplement on the control of prediabetic subjects' glycaemia. Ann Nutr Metab 2020;76 (suppl 4):73.

\section{$451 / 65$}

\section{Effect of Moringa oleifera Leaf on Inflammation Markers and Adipokines in Prediabetic Adult Subjects}

\author{
Díaz-Prieto, L. ' ; Gómez-Martínez, S. ; Heredia-Díaz, C. ${ }^{2}$; \\ Martín-Ridaura, $C^{3}{ }^{\text {; }}$ Vicente, I. ${ }^{1}$; Nova, E. $^{1}$ \\ ${ }^{1}$ Institute of Food Science and Technology and Nutrition \\ (ICTAN)-CSIC; ${ }^{2}$ C.S. Cea Bermúdez, Servicio Madrileño de Salud; \\ ${ }^{3}$ Madrid-Salud, Ayuntamiento de Madrid
}

The aim of this work was to assess whether the leaves of the Moringa oleifera (MO) plant change the plasma inflammatory markers and adipokine concentrations during an intervention study performed in prediabetic adults. A double-blind placebocontrolled, parallel group intervention was conducted ${ }^{(1)}$. Sixty five prediabetic subjects aged between 40 and 75 years finished the study and were included in this analysis (55\% females). Subjects under pharmacological treatment or suffering any serious disease were excluded. Participating subject consumed 6 daily capsules of MO dry leaves powder (400 mg/unit) (MO group, $\mathrm{N}=31$ ) or 6 placebo capsules (PLC group, $\mathrm{N}=34$ ) during 12 weeks. Measures were taken basally (W0), and after 6 (W6) and 12 weeks (W12). Results: No significant interaction "treatment time" was found for any of the biomarkers, however, a significant "time" effect was found for some of them $(\mathrm{P}<0.05)$. This was the case for TNF-alpha (PLC: W0 vs. $\mathrm{W} 12, \mathrm{P}=0.035$; $\mathrm{MO}$ : $\mathrm{W} 6$ vs. $\mathrm{W} 12, \mathrm{P}=0.047)$ and adiponectin (PLC: $\mathrm{W} 0$ vs. $\mathrm{W} 12, \mathrm{P}<0.001 ; \mathrm{MO}$ : $\mathrm{W} 0$ vs. $\mathrm{W} 12, \mathrm{P}<0.001$ ), both, significantly decreasing. IL-6 decreased significantly in MO (W0 vs. $\mathrm{W} 12, \mathrm{P}=0.007)$ and showed only a decreasing trend in PLC (W0 vs. $\mathrm{W} 12, \mathrm{P}=0.090)$. Finally, leptin decreased only in the MO group (W6 vs. W12, $\mathrm{P}=0.010$ ) and IL-1b showed a trends to also decrease in the MO group (W0 vs. W12, $\mathrm{P}=0.082$ ). C-reactive protein and MCP-1 did not change in either group during the intervention. Conclusion: IL- 6 and leptin improved significantly after a 12 week intervention with $\mathrm{MO}$ in prediabetic subjects.

Acknowledgments: The authors are grateful to the volunteers who participated in this study.

Financial Support: Ministerio de Economía, Industria y Competitividad (MINECO), Agencia Estatal de Investigación
(AEI) and Fondo Europeo de Desarrollo Regional (FEDER, UE) (ref. AGL2017-86044-C2-1-R.).

Conflict of Interest: None.

Authorship: SG-M, LD-P, IV and EN performed analytical techniques, $\mathrm{CH}-\mathrm{D}$ and $\mathrm{CM}-\mathrm{R}$ recruited patients, EN designed the study.

Keywords: Moringa oleifera, dietary supplement, prediabetes, cytokines, adipokines.

\section{Reference}

Nova E, Redondo-Useros N, Martínez-García RM, GómezMartínez S, Díaz-Prieto LE, Marcos A. Potential of Moringa oleifera to Improve Glucose Control for the Prevention of Diabetes and Related Metabolic Alterations: A Systematic Review of Animal and Human Studies. Nutrients. 2020 Jul 10;12(7):2050.

\section{$451 / 66$ \\ Do Vegetable Extracts Promote the Growth of Intestinal Bacteria?}

\section{Picón Gávez, A; Rodríguez, E; Vázquez, M}

INIA - Department of Food Technology, Madrid, Spain

The aim of this work was to evaluate the ability of several human intestinal bacteria from baby faeces (Lactobacillus and Bifidobacterium strains belonging to different species) to grow in the presence of polyphenol-rich vegetable extracts. Plant (artichoke, eggplant and moringa) as well as seaweed (Irish moss and sea spaguetti) extracts were selected for their richness in polyphenols (1) and their potential anti-cholesterolemic properties. Lactic acid bacteria, belonging to different species and subspecies, were selected from the INIA bacterial culture collection for their origin (faeces from healthy breast-fed babies) and their ability to withstand in vitro conditions simulating the passage through the gastrointestinal tract (2).

A new and simple method has been developed to screen and compare the effects of vegetable extracts on the growth of the strains. For each specific extract, agar media base plates were supplemented with the extract and aniline blue was added as a redox indicator. Results shown that eggplant and artichoke extracts stimulated the growth of all LAB strains.

Acknowledgments: Projects RTI2018-099271-R-I00 and RTA2017-00002-00.

Financial Support: Project RTI2018-099271-R-I00.

Conflict of Interest: None.

Authorship: Formulating the research questions, APG and ER. Designing the study, APG and ER. Carrying it out, ER, MV and APG. Analysing the data, ER, MV and APG. Writing, ER, MV and APG

Keywords: Vegetable extracts, intestinal bacteria, polyphenols, growth. 


\section{References}

1. Velioglu Y.S., Mazza G., Gao L., Oomah, B.D. (1998). J Agric Food Chem 46, 4113-4117.

2. Rodriguez E. Arques J.L., Rodriguez R., Peiroten A., Landete J.M., Medina M. (2012). J Funct Foods 4, 542-551.

\section{$451 / 67$ \\ 2-hydroxy-(4-methylseleno) Butanoic Induces \\ the Expression of Selenoproteins and Protects against Oxidative Stress in Se-Deprived Macrophages}

Campo-Sabariz, J'; García-Vara, $A^{1}$; Briens, $M^{2}$; Amine-Hachemi, M. ${ }^{2}$; Pinloche, E. ${ }^{2}$; Ferrer, $R{ }^{1}{ }^{1}$; Martín-Venegas, $R^{1}$

${ }^{1}$ Dept. Biochemistry and Physiology, Faculty of Pharmacy and Food Science, University of Barcelona, Spain; INSA-UB; ${ }^{2}$ Adisseo France SAS, Antony, France

The objective was to investigate the role of 2-hydroxy-(4methylseleno) butanoic acid (HMSeBA), an organic form of Se used in animal nutrition, in supporting selenoprotein synthesis and protecting against oxidative stress in macrophages. Inadequate dietary Se compromises selenoprotein synthesis and is considered a risk factor for several chronic diseases associated with oxidative stress. In macrophages, glutathione peroxidase 1 (GPX1) is the most abundant selenoprotein and catalyses the reduction of $\mathrm{H}_{2} \mathrm{O}_{2}$ and organic hydroperoxides. Selenoprotein P (SELENOP) is also highly expressed in macrophages and, although the main function is to deliver Se to the organism, a greater antioxidant activity has been also reported for this selenoprotein (1). GPX1 and SELENOP gene expression, GPX activity, SELENOP protein expression, as well as the production of reactive oxygen species (ROS) were determined as previously described (2) in a model of Se-deprived macrophages (FBS removal, 24h) (3), further supplemented with HMSeBA (12.5 and $625 \mu \mathrm{M}, 72 \mathrm{~h})$. The data indicate that in the Se-deprived macrophages GPX1 and SELENOP gene expression was reduced, being these results confirmed by a reduction in GPX activity and SELENOP protein expression. Se-deprivation also induced an increase in ROS production. Supplementation of these cells with HMSeBA completely restored GPX1 and SELENOP gene expression to the values obtained in the presence of FBS (the main Se source in the culture). Moreover, the results show that in $\mathrm{H} 2 \mathrm{O} 2$-stimulated macrophages, HMSeBA supplementation protects against oxidative stress. In conclusion, HMSeBA supports selenoprotein synthesis in macrophages and contributes to maintaining homeostasis by protecting against oxidative stress.

Acknowledgments: None.

Financial Support: Supported by Adisseo France S.A.S.

Conflict of Interest: None.

Authorship: MB, EP, RF and RM-V designed the research; JC-S and GV-A conducted the research; JC-S and RM-V: analysed the data; JC-S, RM-V and RF edited the abstract and all authors read and approved the final abstract.

Keywords: Macrophages, organic selenium, glutathione peroxidase, selenoprotein $\mathrm{P}$, oxidative stress.

\section{References}

1 Carlson BA, Yoo MH, Sano Y, Sengupta A, Kim JY, Irons R, Gladyshev VN, Hatfield DL, Park JM. Selenoproteins regulate macrophage invasiveness and extracellular matrix-related gene expression. BMC Immunol. 2009 Oct 28; 10:57. doi: 10.1186/1471-2172-10-57.

2 Campo-Sabariz J, Moral-Anter D, Brufau MT, Briens M, Pinloche E, Ferrer R, Martín-Venegas R. 2-Hydroxy-(4-methylseleno) butanoic acid is used by intestinal Caco- 2 cells as a source of selenium and protects against oxidative stress. J Nutr. 2019 Dec 1;149(12):2191-2198. doi: $10.1093 /$ jn/nxz190.

3 Campo-Sabariz J, Moral-Anter D, Brufau MT, Ferrer R, Briens M, Martín-Venegas R. Model of Se deprivation in Caco-2 cells and macrophages in culture. V Jornada de Recerca INSA-UB: "Al-lèrgies i intoleràncies alimentàries: de la sospita a la taula», 2017, http://hdl.handle. net/2445/119055

\section{$451 / 68$}

\section{The Effects of Dietary Patterns on} Immune-Inflammatory Biomarkers: Results from Meta-Analyses of Intervention Studies

\author{
Koelman, L. ${ }^{1}$; Egea Rodrigues, $C^{2}{ }^{2}$; Aleksandrova, $K^{3}$
}

${ }^{1}$ German Institute of Human Nutrition Potsdam-Rehbrücke; ${ }^{2}$ Institute of Pharmacy, Freie Universität Berlin, Berlin, Germany;

${ }^{3}$ Leibniz Institute for Prevention Research and Epidemiology,

Bremen, Germany

Low-grade chronic inflammation is a key player in the pathophysiology of various chronic diseases, including cardiovascular disease, diabetes and cancer [1], as well as for COVID-19 severity [2-4]. This justifies the strong interest in finding strategies to alleviate inflammation and balancing immune system response. Numerous studies explored the role of various dietary patterns in relation to biomarkers of inflammation and immune response; however, the overall evidence has not been summarized. We conducted a systematic review and meta-analysis of randomized controlled trials (RCTs) based on literature search in Pubmed, Medline and Web of Science up to October 2020. Of initially identified 2.497 publications, 22 RCTs met the inclusion criteria [5-26]. Mean differences (MDs) in biomarkers of inflammatory status were estimated for the Mediterranean diet, the Dietary Adherence to Stop Hypertension (DASH) diet, and the vegetarian/vegan diet. The Mediterranean diet showed the most prominent reductions of immune-inflammatory biomarkers, including interleukin-6 (Mean difference (MD): $-1.07 \mathrm{pg} / \mathrm{ml}$ [95\% CI: -1.94, -0.20]; I2: 96\%), interleukin-1b (MD: $-0.46 \mathrm{pg} / \mathrm{ml}$ [95\% CI: $-0.66,-0.25]$; I2: $0 \%$ ) and C-reactive protein (MD: - $1.00 \mathrm{mg} / \mathrm{l}$ [95\% CI: -2.02, 0.01]; I2: $100 \%)$. For the remaining dietary patterns, no substantial differences between baseline and post-intervention measurements were revealed. This meta-analysis highlights that following a Mediterranean diet could represent a valid long-term approach for sustaining immune balance and inflammation control in healthy individuals. Large multifactorial RCTs investigating a larger variety of diets are warranted to allow direct comparison of the effects in future research. 
Acknowledgments: The authors thank Tom Heinze (Institute of Nutritional Sciences, University of Potsdam) for his assistance with literature search and data extraction.

Financial Support: None.

Conflict of Interest: None.

Authorship: KA designed the study; LK conducted the search; LK and CER conducted the data extraction; LK analysed the data. LK, CER and KA drafted the manuscript.

Keywords: Dietary patterns; inflammation; biomarkers; randomized controlled trials; meta-analysis; nutritional interventions.

\section{References}

1 Furman, D., et al., Chronic inflammation in the etiology of disease across the life span. Nat Med, 2019. 25(12): p. 1822-1832.

2 Brodin, P., Immune determinants of COVID-19 disease presentation and severity. Nature Medicine, 2021. 27(1): p. 28-33.

3 Greenberg, A.S. and M.S. Obin, Obesity and the role of adipose tissue in inflammation and metabolism. The American Journal of Clinical Nutrition, 2006. 83(2): p. 461S-465S.

4 Zhou, F., et al., Clinical course and risk factors for mortality of adult inpatients with COVID-19 in Wuhan, China: a retrospective cohort study. Lancet, 2020. 395(10229): p. 1054-1062.

5 Makarewicz-Wujec, M., et al., DASH diet decreases CXCL4 plasma concentration in patients diagnosed with coronary atherosclerotic lesions. Nutrition, Metabolism and Cardiovascular Diseases, 2020. 30(1): p. 56-59.

6 Juraschek, S.P., et al., Associations Between Dietary Patterns and Subclinical Cardiac Injury: An Observational Analysis From the DASH Trial. Ann Intern Med, 2020. 172(12): p. 786-794.

7 Baguley, B.J., et al., Mediterranean-style dietary pattern improves cancer-related fatigue and quality of life in men with prostate cancer treated with androgen deprivation therapy: A pilot randomised control trial. Clin Nutr, 2020.

8 Wade, A.T., et al., Effects of Mediterranean diet supplemented with lean pork on blood pressure and markers of cardiovascular risk: findings from the MedPork trial. Br J Nutr, 2019. 122(8): p. 873-883.

9 Chmurzynska, A., et al., The Effect of Habitual Fat Intake, IL6 Polymorphism, and Different Diet Strategies on Inflammation in Postmenopausal Women with Central Obesity. Nutrients, 2019. 11(7).

10 Mayr, H.L., et al., Randomization to 6-month Mediterranean diet compared with a low-fat diet leads to improvement in Dietary Inflammatory Index scores in patients with coronary heart disease: the AUSMED Heart Trial. Nutr Res, 2018. 55: p. 94-107.

11 Shah, B., et al., Anti-Inflammatory Effects of a Vegan Diet Versus the American Heart Association-Recommended Diet in Coronary Artery Disease Trial. J Am Heart Assoc, 2018. 7(23): p. e011367.

12 Jaacks, L.M., et al., Pilot randomized controlled trial of a Mediterranean diet or diet supplemented with fish oil, walnuts, and grape juice in overweight or obese US adults. BMC Nutr, 2018. 4: p. 26.

13 Wade, A.T., et al., A Mediterranean diet supplemented with dairy foods improves markers of cardiovascular risk: results from the MedDairy randomized controlled trial. Am J Clin Nutr, 2018. 108(6): p. 1166-1182.

14 Duś-Żuchowska, M., et al., The Central European diet as an alternative to the Mediterranean diet in atherosclerosis prevention in postmenopausal obese women with a high risk of metabolic syndrome - a randomized nutrition-al trial. Acta Sci Pol Technol Aliment, 2018. 17(4): p. 399-407.

15 Rallidis, L.S., et al., Short-term effects of Mediterranean-type diet intervention on soluble cellular adhesion molecules in subjects with abdominal obesity. Clin Nutr ESPEN, 2017. 17: p. 38-43.
16 Davis, C.R., et al., A Mediterranean Diet Reduces F(2)-Isoprostanes and Triglycerides among Older Australian Men and Women after 6 Months. J Nutr, 2017. 147(7): p. 1348-1355.

17 Dyer, J., et al., Effect of a Mediterranean Type Diet on Inflammatory and Cartilage Degradation Biomarkers in Patients with Osteoarthritis. J Nutr Health Aging, 2017. 21(5): p. 562-566.

18 Casas, R., et al., Anti-Inflammatory Effects of the Mediterranean Diet in the Early and Late Stages of Atheroma Plaque Development. Mediators Inflamm, 2017. 2017: p. 3674390.

19 Monfort-Pires, M. and S.R.G. Ferreira, Inflammatory and metabolic responses to dietary intervention differ among individuals at distinct cardiometabolic risk levels. Nutrition, 2017. 33: p. 331-337.

20 Maiorino, M.I., et al., Mediterranean diet cools down the inflammatory milieu in type 2 diabetes: the MÉDITA randomized controlled trial. Endocrine, 2016. 54(3): p. 634-641.

21 Razavi Zade, M., et al., The effects of DASH diet on weight loss and metabolic status in adults with non-alcoholic fatty liver disease: a randomized clinical trial. Liver Int, 2016. 36(4): p. 563-71.

22 Casas, R., et al., Long-Term Immunomodulatory Effects of a Mediterranean Diet in Adults at High Risk of Cardiovascular Disease in the PREvención con DIeta MEDiterránea (PREDIMED) Randomized Controlled Trial. J Nutr, 2016. 146(9): p. 1684-93.

23 Gomez-Delgado, F., et al., Chronic consumption of a low-fat diet improves cardiometabolic risk factors according to the CLOCK gene in patients with coronary heart disease. Mol Nutr Food Res, 2015. 59(12): p. 2556-64.

24 Asemi, Z. and A. Esmaillzadeh, DASH diet, insulin resistance, and serum hs-CRP in polycystic ovary syndrome: a randomized controlled clinical trial. Horm Metab Res, 2015. 47(3): p. 232-8.

25 Fritzen, A.M., et al., New Nordic Diet-Induced Weight Loss Is Accompanied by Changes in Metabolism and AMPK Signaling in Adipose Tissue. J Clin Endocrinol Metab, 2015. 100(9): p. 3509-19.

26 Adamsson, V., et al., Role of a prudent breakfast in improving cardiometabolic risk factors in subjects with hypercholesterolemia: a randomized controlled trial. Clin Nutr, 2015. 34(1): p. 20-6.

\section{$451 / 69$ \\ Comparative Analysis of Nutrient Intake Status in Autoimmune Diseases versus Healthy Subjects}

\author{
Rivera Escoto, M.; Meza-Meza, M.; Ruiz-Ballesteros, A.; \\ Pésquela-Cendejas, K.; Mora-García, P.; de la Cruz Mosso, U. \\ Immunonutrition and Nutrigenomics Group of Autoimmune \\ Diseases, University of Guadalajara, Mexico
}

Rheumatoid arthritis (RA) and systemic lupus erythematosus (SLE) are autoimmune inflammatory diseases (1-4), where unhealthy dietary patterns have been linked to exacerbation of clinical symptoms and comorbidities development (4-7). The excessive consumption of calories, simple carbohydrates, saturated fats, and sodium have been related to a worse prognosis, whereas omega- 3 and vitamin D are related to an anti-inflammatory status $(4,5,8-10)$. The aim of this study was to compare the dietary intake of patients with RA $(n=71)$, SLE $(n=71)$ versus Healthy subjects (HS) $(n=71)$ to identify consumption deficiencies according to the dietary reference intake (DRI) established for Mexican population. We found that RA and SLE patients consume more carbohydrates and lipids $(\mathrm{RA}=54.70 \%, \mathrm{SLE}=53.01 \%$ vs. $\mathrm{HS}=45.25 \%)$, also they have less consume of vitamin $\mathrm{D},(\mathrm{AR}=3.22$, $\mathrm{SLE}=3.07 \mathrm{vs} . \mathrm{HS}=4.17 \mu \mathrm{g} /$ day $)$, vitamin $\mathrm{E},(\mathrm{RA}=0.83, \mathrm{SLE}=0.99 \mathrm{vs}$. 
$\mathrm{HS}=2.46 \mu \mathrm{g} /$ day $)$, selenium, $(\mathrm{RA}=74.39, \mathrm{SLE}=69.15$ vs. $\mathrm{HS}=88.68$ $\mu \mathrm{g}$ /day) than HS. According to DRI, $44 \%$ of AR patients have an excessive consume of calories and $52 \%$ of lipids vs. HS $(\mathrm{p}<0.01)$, $43 \%$ of SLE patients show a deficient consumption of calories, $41 \%$ of protein ${ }^{*}, 43 \%$ of lipids vs HS ( $\left.p<0.01\right)$, and the HS presented deficient intakes of micronutrients (vitamin D, E) according to the DRI. Moreover, we found negative correlations between zinc intake with $\mathrm{C}$-reactive protein $(\mathrm{r}=-0.357, \mathrm{p}<0.05)$, and selenium intake with VSG $(r=-0.390, p<0.05)$. In conclusion, nutrient intake deficiencies are more frequent in patients with RA and SLE compared to HS, and micronutrients correlate with inflammatory markers in autoimmunity.

Acknowledgments: University of Guadalajara, UdeG.

Financial Support: The authors acknowledge the support of the grant PRO-SNI 2017-2020 to Ulises de la Cruz-Mosso, from the Programa de Apoyo a la Mejora en las Condiciones de Producción de los Miembros del SNI y SNCA 2017-2020 of the University of Guadalajara.

Conflict of Interest: None. The authors declare that they have no conflict of interest or competing for financial interest.

Authorship: MRE: research question design, sample capture, nutritional assessment, data analysis, article writing. MM-M, AR-B, KP-C: sample capture, nutritional assessment, blood sample processing and biochemical analysis. PM-G: data processing, dietary assessement. UdCM: direction and management of the research project.

Keywords: Rheumatoid arthritis, Systemic Lupus Erythematosus, nutritional status, nutrients intake, dietary assessment.

\section{References}

1. Smolen JS, Aletaha D, Barton A, Burmester GR, Emery P, Firestein GS, et al. Rheumatoid arthritis. Nat Rev Dis Primer. 2018 Jun 7;4(1):18001.

2. Tsokos GC. Systemic Lupus Erythematosus. N Engl J Med. 2011;12.

3. Ahn SS, Jung SM, Song JJ, Park Y-B, Lee S-W. Prognostic nutritional index is correlated with disease activity in patients with systemic lupus erythematosus. Lupus. 2018 Sep;27(10):1697-705.

4. Hayashi H, Satoi K, Sato-Mito N, Kaburagi T, Yoshino H, Higaki M, et al. Nutritional status in relation to adipokines and oxidative stress is associated with disease activity in patients with rheumatoid arthritis. Nutrition. 2012 Nov;28(11-12):1109-14.

5. Masuko K. A Potential Benefit of "Balanced Diet" for Rheumatoid Arthritis. Front Med. 2018 May 15;5:141.

6. Meza-Meza MR, Vizmanos-Lamotte B, Muñoz-Valle JF, Parra-Rojas I, Garaulet M, Campos-López B, et al. Relationship of Excess Weight with Clinical Activity and Dietary Intake Deficiencies in Systemic Lupus Erythematosus Patients. Nutrients. 2019 Nov 6;11(11):2683.

7. Scrivo R, Perricone C, Altobelli A, Castellani C, Tinti L, Conti F, et al. Dietary Habits Bursting into the Complex Pathogenesis of Autoimmune Diseases: The Emerging Role of Salt from Experimental and Clinical Studies. Nutrients. 2019 May 5;11(5):1013.

8. Alunno A, Nikiphorou E, Philippou E, Daien C, Wiek D, Kouloumas M, et al. Nutrition in RMDs: is it really food for thought? Focus on rheumatoid arthritis. BMC Rheumatol. 2020 Dec;4(1):10.

9. Aparicio-Soto M, Sánchez-Hidalgo M, Alarcón-de-la-Lastra C. An update on diet and nutritional factors in systemic lupus erythematosus management. Nutr Res Rev. 2017 Jun;30(1):118-37.

10. La Cava A. The Influence of Diet and Obesity on Gene Expression in SLE. Genes. 2019 May 27;10(5):405.

\section{$451 / 70$ \\ Association of High Calcitriol with Clinical/Renal Activity in Vitamin D Serum Deficient-Systemic Lupus Erythematosus Patients}

Meza-Meza, M. ${ }^{1}$; Ruiz-Ballesteros, A. ${ }^{1}$; Vizmanos-Lamotte, B. ${ }^{2}$; Parra-Rojas, I. ; Muñoz-Valle, J. ${ }^{4}$; de la Cruz Mosso, U. ${ }^{1}$

${ }^{1}$ Immunonutrition and Nutrigenomics Group of Autoimmune Diseases, University of Guadalajara, Mexico; ${ }^{2}$ Institute of Nutrigenetics and Nutrigenomics, University of Guadalajara, Mexico; ${ }^{3}$ Research Laboratory of Obesity and Diabetes, Faculty of Chemical-Biological Sciences, University of Guerrero, Mexico; ${ }^{4}$ Institute of Biomedical Sciences, University of Guerrero, Mexico

Vitamin D (calcidiol) deficiency in systemic lupus erythematosus (SLE) is more frequent than healthy subjects (HS); it has been associated with SLE clinical disease activity and damage, with controversial findings. Although calcidiol is considered the best indicator of vitamin D serum status, its deficiency could not reflect the serum status and conversion of its active metabolite, calcitriol. This study was aimed to assess the association of calcidiol, calcitriol, and calcitriol/calcidiol ratio with clinical and renal disease activity in SLE patients. A cross-sectional study was conducted in 228 SLE and HS Mexican-mestizo women; calcidiol and calcitriol serum levels were evaluated by immunoassays. SLE patients showed lower serum calcidiol $(21.2 \mathrm{ng} / \mathrm{mL})$ and higher calcitriol $(46.8 \mathrm{pg} / \mathrm{mL})$ than HS $(24.5 \mathrm{ng} / \mathrm{mL}$ and $38.1 \mathrm{pg} / \mathrm{mL})$. SLE patients with clinical and renal disease activity had higher frequency of calcidiol deficiency ( $48 \%$ and $52 \%$, respectively) with a pattern of higher calcitriol serum levels $(48.4 \mathrm{pg} / \mathrm{mL}$ and $47 \mathrm{pg} / \mathrm{mL}$, respectively) and higher calcidiol to calcitriol conversion rate $(2.78 \mathrm{pg} /$ $\mathrm{ng}$ and $2.13 \mathrm{pg} / \mathrm{ng}$ respectively) than HS (1.46 pg/ng). In SLE, calcidiol was negatively correlated with calcitriol $(\mathrm{r}=-0.26 ; \mathrm{p}=0.001)$ and urine proteins $(\mathrm{r}=-0.39 ; \mathrm{p}<0.01)$ while calcitriol was positively correlated with blood lymphocytes $(\mathrm{r}=0.30 ; \mathrm{p}<0.001)$, and negatively correlated with the glomerular filtration rate $(r=-0.28$; $\mathrm{p}=0.001)$. High calcitriol levels were associated with risk to clinical disease activity $(\mathrm{OR}=3.31 ; 95 \% \mathrm{CI}=1.27-8.73$; $\mathrm{p}<0.01)$. In conclusion, a pattern of high calcitriol serum levels and a calcidiol to calcitriol conversion rate was observed in clinical and renal active SLE patients with vitamin D deficiency.

Acknowledgments: To the institutions that provided financial and structural support for the project, and to all members of the Grupo de Inmunonutrición y Genómica Nutricional en las Enfermedades Autoinmunes, Centro Universitario de Ciencias de la Salud; Universidad de Guadalajara.

Financial Support: This study was supported by Grant No. UDG-PTC 1401 for Ulises de la Cruz-Mosso, PhD (UDCM), from the Apoyo a la Incorporación de NPTC SEP-PRODEP 2018, and in part by the Grant PRO-SNI 2017-2020 (UDCM) Programa de Apoyo a la Mejora en las Condiciones de Producción de los Miembros del SNI y SNCA 2017-2019 from the University of Guadalajara. The funding sources were not involved in any step of the study.

Conflict of Interest: The authors declare that the research was conducted in the absence of any commercial or financial relationships that could be construed as a potential conflict of interest. All authors approved the final version of the manuscript. 
Authorship: Conceptualization, U.D.C.M.; methodology, MM-M, AR-B, BV-L, JM-V; formal analysis, MM-M, IP-R; investigation, MM-M, AR-B; resources, U.D.C.M; writing-original draft preparation, MM-M; writing-review and editing, U.D.C.M; AR-B, BV-L, JM-V, IP-R; visualization, U.D.C.M; supervision, U.D.C.M; project administration, U.D.C.M; funding acquisition, U.D.C.M. All authors have read and agreed to the published version of the manuscript.

Keywords: Calcitriol, calcidiol deficiency, clinical/renal activity, SLE, vitamin D hydroxylation.

\section{References}

Azrielant, S.; Shoenfeld, Y. Eppur Si Muove: vitamin D is essential in preventing and modulating SLE. Lupus. 2016, 25, 563-572, doi.org/10.1177/ 0961203316629001.

Eloi, M.; Horvath, D.V.; Ortega, J.C.; Prado, M.S.; Andrade, L.E.C.; Szejnfeld, V.L.; et al. 25-Hydroxivitamin D Serum Concentration, Not Free and Bioavailable Vitamin D, Is Associated with Disease Activity in Systemic Lupus Erythematosus Patients. PLoS One. 2017, 12, doi.org/10.1371/ journal.pone.0170323.
Gao, C.C.; Liu, S.Y.; Wu, Z.Z.; Li, T.F.; Gao, G.M.; Liu, Z.S.; et al. Severe vitamin D deficiency increases the risk for moderate to severe disease activity in Chinese patients with SLE. Lupus. 2016, 25, 1224-1229, doi. org/10.1177/0961203316635289.

García-Carrasco, M.; Mendoza-Pinto, C.; Etchegaray-Morales, I.; Soto-Santillán, P.; Jiménez-Herrera, E.A.; Robles-Sánchez, V.; et al. Vitamin D insufficiency and deficiency in mexican patients with systemic lupus erythematosus: Prevalence and relationship with disease activity. Reumatol Clin. 2017, 13, 97-101, doi.org/10.1016/j.reuma.2016.02.013.

Meza-Meza, M.R.; Ruiz-Ballesteros, A.I.; De la Cruz-Mosso, U. Functional effects of vitamin D: From nutrient to immunomodulator. Crit Rev Food Sci Nutr. 2020, 0, 1-21, doi.org/10.1080/10408398.2020.1862753.

Ong S.G.; Ding, H.J. Vitamin D status in a monocentric cohort of systemic lupus erythematosus (SLE) patients and correlations with clinical and immunological profile. Med J Malaysia. 2019, 74, 6, 492-498.

Pasquali, M.; Tartaglione, L.; Rotondi, S.; Muci, M.L.; Mandanici, G.; Farcomeni, A.; et al. Calcitriol/calcifediol ratio: An indicator of vitamin D hydroxylation efficiency? BBA Clinical. 2015, 3, 251-256, doi.org/10.1016/j. bbacli.2015.03.004.

Shoenfeld, Y.; Giacomelli, R.; Azrielant, S.; Berardicurti, O.; Reynolds, J.A.; Bruce, I.N. Vitamin D and systemic lupus erythematosus - The hype and the hope. Autoimmun Rev. 2018, 17, 19-23, doi.org/10.1016/j.autrev.2017.11.004. 


\section{Author Index}

\section{Nutrition\& Metabolism}

Aleksandrova, K. 451/68

Alonso-Lovera, P. 451/33

Amat, C. 451/37, 451/38

Amine-Hachemi, M. 451/67

Andrés-Lacueva, C. 451/49

Angel-Isaza, J. 451/10, 451/25

Ayechu-Muruzabal, V. 451/11

Azagra Boronat, I. 451/30, $451 / 53,451 / 54$

Badía Palacín, J. 451/41

Baldomà Llavinés, L. 451/41

Benavente, F. 451/50

Benharrats, L. 451/14, $451 / 23$

Berezovski, M. 451/50

Best, I. 451/17, 451/55

Blanco Rogel, M. 451/45

Bouzas Muñoz, A. 451/48

Briens, M. 451/67

Calle, N. 451/64

Campo-Sabariz, J. 451/67

Candiloro, F. 451/9

Capdevila Menac, N. 451/45

Cardelle-Cobas, A. 451/33

Cardona Burrull, F. 451/45

Carmona Hernandez, J. $451 / 10,451 / 25$

Carrillo-Vico, A. 451/16

Castell Escuer, M. 451/17, $451 / 35,451 / 54,451 / 55$, $451 / 56$

Cepeda Sáez, A. 451/33

Ceprian, N. 451/43, $451 / 44$

Chen, H. 451/12

Childs, C. 451/28

Collado, M. 451/53

Cordero Alday, C. 451/41

Cruz Chamorro, I. 451/16

de la Cruz Mosso, U. 451/69, 451/70

de la Fuente, M. 451/43, 451/44

Del Fabbro, S. 451/28

Díaz, L. 451/58, 451/64

Diaz, N. 451/41
Numbers refer to abstract numbers
Diaz-Del Cerro, E. 451/43, 451/44

Díaz-Prieto, L. 451/60, 451/65

Drenjancevic, I. 451/32, $451 / 34,451 / 36,451 / 42$

Egea Rodrigues, C. 451/68

Fanning, A. 451/19

Feliu, M. 451/57

Fernandez, I. 451/57

Fernández-Santos, J. 451/16

Fernández-Tomé, S. 451/26

Ferrer, R. 451/67

Franch Masferrer, Á. 451/35, 451/54

Franco-Ávila, T. 451/59

Galindo Luján, R. 451/50

Gallego Vendrell, E. 451/52

Garcia Tejedor, A. 451/39

Garcia-Solis, B. 451/43

Garssen, J. 451/11, 451/12, $451 / 30,451 / 31$

Gimenez-Llort, L. 451/43

Godoy, M. 451/58

Gómez Contreras, A. 451/59

Gomez de Cedron, M. 451/48

Gómez Martínez, S. 451/60, 451/61, 451/62, 451/64, $451 / 65$

González-Correa, C. 451/10, $451 / 25$

Gonzalez Zancada, N. 451/60

González-Romero, E. 451/64

Grases-Pintó, B. 451/35, $451 / 63$

Guadarrama-López, A. $451 / 40$

Gueimonde, M. 451/44

Gutierrez-Hervas, A. 451/61, $451 / 62$

Harbige, L. 451/51

Harrington, D. 451/51

Heredia-Díaz, C. 451/65

Hernández-Ledesma, B. 451/26

Hogenkamp, A. 451/12
Indiano-Romacho, P. 451/26

Insani, E. 451/58

Izquierdo-Gómez, R. 451/61, $451 / 62$

Juan, M. 451/59

Jukic, I. 451/32

Knezović, A. 451/42

Knippels, L. 451/12

Knipping, K. 451/30, 451/31

Koelman, L. 451/68

Kolobarić, N. 451/34, 451/36

Kostadinova, A. 451/11

Kozina, N. 451/32

Lambea, M. 451/44

Laparra Llopis, J. 451/39, $451 / 48$

MacMahon, E. 451/51

Malczewska-Lenczowska, J. $451 / 46$

Mambrin, M. 451/57

Marcos, A. 451/58, 451/60, $451 / 61,451 / 62,451 / 64$

Márquez Contreras, E. 451/59

Martín-Ridaura, C. 451/65

Martín-Venegas, R. 451/67

Martínez-Carrillo, B. 451/40

Massot-Cladera, M. 451/17, 451/31, 451/54, 451/55

Matić, A. 451/34

Mayordomo-Cava, J. 451/49

Mekki, K. 451/14, 451/23

Melgar, S. 451/19

Meza-Meza, M. 451/69, $451 / 70$

Mihalj, M. 451/34, 451/42

Mihaljević, Z. 451/36, 451/42

Millán-Linares, M. 451/16

Minic, Z. 451/50

Miró, L. 451/37, 451/38, $451 / 59$

Młynarczyk, M. 451/46

Mora-García, P. 451/69

Morales-Ferré, C. 451/30, $451 / 31,451 / 63$

Morant, L. 451/52
Moretó, M. 451/37, 451/38, $451 / 59$

Muñoz-Valle, J. 451/70

Narváez Solarte, W. 451/25

Nova, E. 451/58, 451/60, $451 / 64,451 / 65$

Olivo, Y. 451/41

Orysiak, J. 451/46

Overbeek, S. 451/11

Parra-Rojas, I. 451/70

Pastor-Soplin, S. 451/17, 451/55

Paterson Moreno, S. 451/26

Pedroche, J. 451/16

Pérez-Bosque, A. 451/37, 451/38

Pérez-Cano, F. $\quad 451 / 17$, 451/30, 451/31, 451/35, $451 / 53,451 / 54,451 / 55$, $451 / 56,451 / 63$

Périz, M. 451/17, 451/55, $451 / 56$

Perris, P. 451/57

Pésquela-Cendejas, K. 451/69

Picón Gálvez, A. 451/66

Pinloche, E. 451/67

Planas, J. 451/59

Polo, J. 451/37, 451/38

Pont, L. 451/50

Ramírez, A. 451/48

Redondo Useros, N. 451/60

Reglero, G. 451/48

Restrepo López, J. 451/10, $451 / 25$

Rio Aige, K. 451/53, 451/56

Rivera Escoto, M. 451/69

Rodríguez, E. 451/66

Rodríguez-Lagunas, M. 451/53, 451/30, 451/31, $451 / 35,451 / 56,451 / 63$

Román Casas, M. 451/40

Rosell Cardona, C. 451/37, $451 / 38$ 
Ruiz-Ballesteros, A. 451/69, 451/70

Ruiz-Iglesias, P. 451/54, $451 / 56$

Saiz Gonzalo, G. 451/19

Salazar Garzo, N. 451/44

Santos Sánchez, G. 451/16

Sanz-Nebot, V. 451/50

Selma Royo, M. 451/53

Senouci, A. 451/14, 451/23

Serra-Rexach, J. 451/49
Shankar-Hari, M. 451/51

Sinisterra Loaiza, L. 451/33

Sirvent Segura, E. 451/52

Slobodianik, N. 451/57, $451 / 58$

Sobczynska-Malefora, A. $451 / 51$

Soriano-Romaní, L. $451 / 52$

Steed, D. $451 / 51$

Stupin, A. 451/36, 451/42

Surała, O. 451/46
Šušnjara, P. 451/34, 451/36, $451 / 42$

Szczepańska, B. 451/46

Toldi, G. 451/24

Tomás Cobos, L. 451/52

Tor Roca, A. 451/49

Urpí-Sardà, M. 451/49

Valdés-Ramos, R. 451/40

Vallverdú-Queralt, A. 451/63 van't Land, B. 451/11

Vázquez Belda, B. 451/33

Veiga, Ó. 451/61, 451/62

Viadel Crespo, B. 451/52

Vicente, I. 451/65

Vizmanos-Lamotte, B. $451 / 70$

Willemsen, L. 451/11

Zhan-Dai, S. 451/63

Zhao, X. 451/12 